NASA Contractor Report 195297

$1 N-02$

9994

$p-51$

\title{
Installed F/A-18 Inlet Flow Calculations at 30 Degrees Angle-of-Attack: A Comparative Study
}

C. Frederic Smith and Steve D. Podleski

Sverdrup Technology, Inc.

Lewis Research Center Group

Brook Park, Ohio

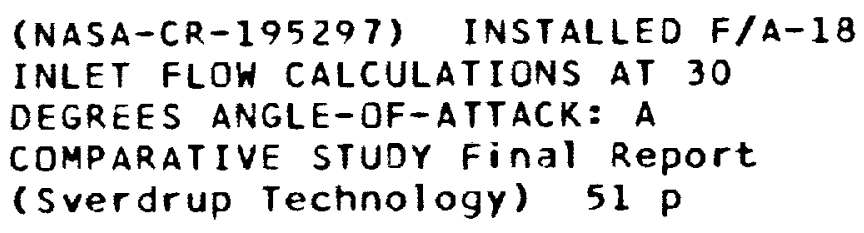

N94-33119

Unclas

G3/02 0009994

Prepared for

Lewis Research Center

Under Contract NAS3-25266

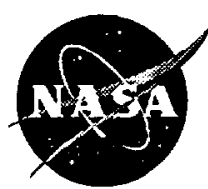

National Aeronautics and Space Administration 



\title{
Installed F/A-18 Inlet Flow Calculations at 30 Degrees Angle-of-Attack: A Comparative Study
}

\author{
C. Frederic Smith and Steve D. Podleski \\ Sverdrup Technology, Inc. \\ Lewis Research Center Group \\ Brook Park, Ohio 44142
}




\section{TABLE OF CONTENTS}

Page

Table of Contents $\ldots \ldots \ldots \ldots \ldots$ ii

List of Figures . . . . . . . . . . . . . . . . . iii

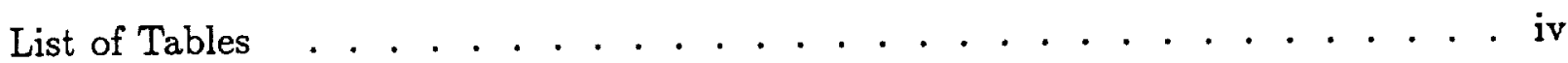

Nomenclature . . . . . . . . . . . . . . . . v v

Summary ........................ . . . . . . .

1.0 Introduction . . . . . . . . . . . . . . . . 1

2.0 Experimental Program . . . . . . . . . . . . . . 4

3.0 Numerical Modelling . . . . . . . . . . . . . . . . . 5

3.1 PARC3D Code . . . . . . . . . . . . . . . . . 5

3.2 CFL3D Code . . . . . . . . . . . . . . . 5

3.3 Grid Generation . . . . . . . . . . . . . . 6

3.4 Boundary Conditions . . . . . . . . . . . . . . 9

3.4.1 PARC3D Code . . . . . . . . . . . . . . . 9

3.4 .2 CFL3D Code . . . . . . . . . . . . . . . . . . . 9

4.0 Results . . . . . . . . . . . . . . . 11

4.1 External Flow Field . . . . . . . . . . . . . . . . 11

4.2 Inlet Duct Flow Field . . . . . . . . . . . . . . . 13

4.3 Numerical Issues . . . . . . . . . . . . . . . . . 18

5.0 Conclusions and Recommendations . . . . . . . . . . 19

6.0 Acknowledgements . . . . . . . . . . . . . . 21

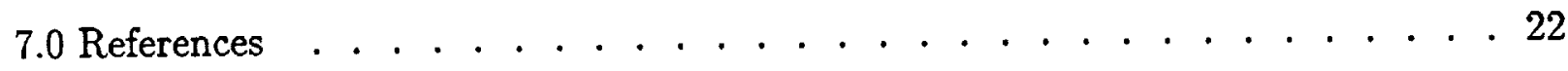




\section{LIST OF FIGURES}

1.

2 .

3.

4.

5 .

6.

7.

8.

9.

10.

\section{1.}

12.

13.

14.

15.

16. Inlet Exit Vorticity Contours $\underline{\text { Page }}$

Particle Trajectories Along Surface:

a. Original Grid; b. Revised Grid; c. CFL3D Grid

Particle Trajectories Ahead of Ramp:

a. Original Grid; b. Revised Grid; c. CFL3D Grid

32

Particle Trajectories Along Ramp:

a. Original Grid; b. Revised Grid; c. CFL3D Grid

33

Forebody/LEX Pressure Measurement Stations

Forebody/LEX Surface Static Pressure Distributions

35

37

Inlet Entrance Total Pressure Contours

a. Original Grid; b. Revised Grid; c. CFL3D Grid

Inlet Exit Total Pressure Contours

a. Original Grid; b. Revised Grid; c. CFL3D Grid

a. Average distortion; b. Peak distortion 


\section{LIST OF TABLES}

\section{Page}

1. Inlet Performance Summary . . . . . . . . . . . . . . . . . . . 24

2. Code Performance Summary . . . . . . . . . . . . . . . . . 24 
Nomenclature

\begin{tabular}{|c|c|c|}
\hline $\mathrm{C}_{p}$ & $=$ & $\begin{array}{l}\left(\mathrm{P}-\mathrm{P}_{\infty}\right) / 1 / 2 \rho_{\infty} \mathrm{V}_{\infty}^{2} \\
\text { (Static Pressure Coefficient) }\end{array}$ \\
\hline Corrected flow rate & $=$ & $(\dot{m} \sqrt{\theta}) / \delta$ \\
\hline Distortion & $=$ & $\begin{array}{l}\left(\mathrm{P}_{T \max }-\mathrm{P}_{T \min }\right) / \mathrm{P}_{T_{\text {avg }}} \text { at } \\
\text { compressor face }\end{array}$ \\
\hline FS & $=$ & $\begin{array}{l}\text { Fuselage Station in Full Scale inches } \\
\text { (FS=0 located } 60.5 \text { inches ahead } \\
\text { of nose) }\end{array}$ \\
\hline$\dot{\mathrm{m}}$ & $=$ & Mass flow rate \\
\hline $\mathrm{P}$ & $=$ & Local static pressure \\
\hline$P_{\infty}$ & $=$ & Free-stream static pressure \\
\hline $\mathrm{P}_{T}$ & $=$ & Local total pressure \\
\hline$P_{\text {Tavg }}$ & $=$ & Average total pressure \\
\hline$P_{T \infty}$ & $=$ & Free stream total pressure \\
\hline$P_{T \min }$ & $=$ & Minimum total pressure \\
\hline $\mathrm{P}_{T_{\max }}$ & $=$ & Maximum total pressure \\
\hline Recovery & $=$ & $\begin{array}{l}\text { Average ratio of } \mathrm{P}_{T} / \mathrm{P}_{T \infty} \\
\text { from } 40 \text { probe rake }\end{array}$ \\
\hline $\mathrm{T}_{\text {Tavg }}$ & $=$ & Average total temperature \\
\hline $\mathrm{V}_{\infty}$ & $=$ & Free-stream velocity \\
\hline$\alpha$ & $=$ & Angle of attack \\
\hline$\beta$ & $=$ & Angle of yaw \\
\hline$\delta$ & $=$ & $\mathrm{P}_{\text {Tavg }} /\left(14.696 \mathrm{lb} / \mathrm{ft}^{2}\right)$ \\
\hline$\rho_{\infty}$ & $=$ & Free-stream density \\
\hline$\theta$ & $=$ & $\mathrm{T}_{T_{\text {avg }}} / 519.0^{\circ} \mathrm{R}$ at compressor face \\
\hline
\end{tabular}




\section{Summary}

NASA Lewis is currently engaged in a research effort as a team member of the High $\mathrm{Al}$ pha Technology Program (HATP) within NASA. This program utilizes a specially equipped F/A-18, the High Alpha Research Vehicle (HARV), in an ambitious effort to improve the maneuverability of high-performance military aircraft at low- subsonic-speed, high angleof-attack conditions. The overall objective of the Lewis effort is to develop inlet technology that will ensure efficient airflow delivery to the engine during these maneuvers. One part of the Lewis approach utilizes computational fluid dynamics codes to predict the installed performance of inlets for these highly maneuverable aircraft.

Full Navier-Stokes (FNS) calculations on the installed F/A-18 inlet at 30 degrees angle of attack, 0 degrees yaw and a freestream Mach number of 0.2 have been obtained in this study using an algebraic turbulence model with two grids (original and revised). Results obtained with the original grid were used to determine where further grid refinements and additional geometry were needed. In order to account properly for the external effects, the forebody, Leading Edge Extension (LEX), ramp, and wing were included with inlet geometry. In the original grid, the diverter, LEX slot and leading edge flap were not included due to insufficient geometry definition. These were included in a revised grid and the results obtained with both configurations are presented in this report. In addition, a thin-layer Navier-Stokes (TLNS) code is used with the revised grid and the numerical results are compared to those obtained with the FNS code. The TLNS code was used to evaluate the effects on the solution using a code with more recent CFD developments included such as upwinding with TVD schemes versus central differencing with artificial dissipation. The calculations are compared to a limited amount of available experimental data. The predicted forebody/fuselage surface static pressures compared well with data for all solutions. The predicted trajectory of the vortex generated under the LEX was different for each solution. These discrepancies are attributed to differences in the grid resolution and turbulence modelling. All solutions predict that this vortex is ingested by the inlet. The predicted inlet total pressure recoveries are lower than data and the distortions are higher than data. The results obtained with the revised grid were significantly improved from the original grid results. The original grid results indicated the ingested vortex migrated to the engine face and caused additional distortions to those already present due 
to secondary flow development. The revised grid results indicate that the ingested vortex is dissipated along the inlet duct inboard wall. The TLNS results indicate the flow at the engine face was much more distorted than the FNS results and is attributed to the pole boundary condition introducing numerical distortions into the flow field. 


\subsection{Introduction}

NASA Lewis is currently engaged in a research effort as a team member of the High Alpha Technology Program (HATP) within NASA. This program utilizes a specially equipped F/A-18, the High Alpha Research Vehicle (HARV), in an ambitious effort to improve the maneuverability of high-performance military aircraft at low-subsonic-speed, high angleof-attack conditions. The overall objective of the Lewis effort is to develop inlet technology that will ensure efficient airflow delivery to the engine during these maneuvers. One part of the Lewis approach utilizes computational fluid dynamics codes to predict the installed performance of inlets for these highly maneuverable aircraft.

One of the goals of the NASA high alpha technology program is to accurately predict the aerodynamics of aircraft operating at extreme attitudes (alpha $=60 \mathrm{deg}$, beta $=$ $10 \mathrm{deg}$ ). As part of this program, NASA Ames-Dryden, Ames-Moffett and Langley are concentrating on external aerodynamics including thrust vectoring control systems and vortex flow control. NASA Lewis is studying the effects of high angle-of-attack and yaw flight conditions on flow within the F/A-18 inlet duct. Details of this cooperative program are contained in Ref. 1. The effects of rate of change in the angle of attack and yaw will be considered for the flight tests only.

The F/A-18 aircraft has experienced engine stalls at high angles of attack and yaw flight conditions which were outside of the flight envelope. At these flight conditions, high angular rates were also present. Future fighter aircraft will be designed to operate routinely in this flight regime. Therefore, it is essential that an understanding of the inlet flow field at these flight conditions be obtained. Due to the complex interactions of the fuselage flow field and the inlet flow field, a study of the flow within the inlet must also include the external effects. Past calculations of flow about the F/A-18 have not included the inlet and ramp [Ref. 2,3]. These features are usually fared over and assumed not to influence the external flow field significantly. However, the effects from the upstream forebody, Leading Edge Extension (LEX) and diverter must be included in order to provide the proper inflow conditions to the inlet duct.

The results of including the inlet and ramp in the flow simulation are reported in Ref. 4. The results of this prior effort formed the foundation for the work that is reported in this report. The solutions obtained in this previous study were evaluated and several short- 
comings were identified. This resulted in obtaining a much more detailed and complete geometry data base of the F/A-18 aircraft and inlet. In addition several areas of the computational grid were revised to improve flow field and surface resolution. Also, the total number of grid points used to model additional components in the revised grid increased only approximately $10 \%$. This was accomplished by reducing the number of grid points in regions where the flow field did not appear to have a significant impact on the inlet flow conditions.

Full Navier-Stokes calculations on the installed F/A-18 inlet at 30 degrees angle of attack, 0 degrees yaw and a freestream Mach number of 0.2 have been obtained in this study using the PARC3D code [Ref. 5] with an algebraic turbulence model. In order to account properly for the external effects, the forebody, LEX, ramp, and wing were included with inlet geometry as shown in Figure 1a. In the original study, the diverter, LEX slot and leading edge flap were not included due to insufficient geometry definition. These were included in the revised geometry as shown in Figure $1 \mathrm{~b}$ and the results obtained with both configurations are presented in this report. Results obtained with the original grid were used to determine where further grid refinements and additional geometry were needed. The calculations are compared to data that presently exist (Ref. 6,7). In particular, data for the inlet flow field is very limited. These results will be more fully compared with data obtained from a $19.78 \%$ scale forebody/inlet model of the F/A-18 aircraft which is scheduled to be tested in 1995 at NASA Lewis and also with flight test data from the HARV in 1994. The purpose of these comparisons is to evaluate/validate the codes. The codes will also be used to aid in the interpretation of the experimental results. One of the objectives of the CFD effort is to aid in the interpretation of the experimental results. The numerical simulations can provide information concerning regions of the flow field that are not measured.

In addition, a comparison of the PARC3D results was made with those obtained using another code. The code chosen was CFL3D [Ref. 8] which has been used extensively for external flow calculations at NASA Langley. The TLNS code was used to evaluate the effects on the solution using a code with more recent CFD developments included such as upwinding with TVD schemes versus central differencing with artificial dissipation. A few modifications were made to the code to accommodate the required internal flow boundary 
conditions and grid topologies. Also, a modified version of the revised grid was used. The major differences in grid topologies were the deletion of the embedded $\mathrm{C}$-grid around the inlet highlight and the flap because the version of CFL3D used could not accommodate these overlaid grids.

In addition to comparing the CFL3D and PARC3D solutions, some of the strengths and weaknesses of the two codes could be compared when they are applied to a complicated configuration such as the F/A-18 forebody/inlet geometry. However, this is not considered to be an exhaustive comparison of the two codes.

This report is divided into several sections. A brief description of the proposed flight and model test programs is presented. This is followed by a discussion of the numerical modelling of this problem which includes a brief description of the flow analysis codes used, the grid generation process and the boundary conditions posed. A comparison of the numerical results obtained for the external flow field features are presented along with a comparison of the predicted and measured surface static pressures along the forebody and LEX. The numerical results for the inlet flow field are then presented along with a comparison to experimental total pressure contours and performance data. A short discussion of various numerical issues raised during this study is presented. Major conclusions and recommendations conclude the report. 


\subsection{Experimental Program}

An experimental program to study the inlet duct flow is planned at Lewis. A $19.78 \%$ scale forebody/inlet model of the F/A-18 aircraft is scheduled to be tested in the Lewis $9 \times 15$ wind tunnel. Details of the airframe aft of the engine face plane will not be included. Measurements are planned to include steady and dynamic total pressure surveys at various stations within the inlet, including a 40 probe total pressure rake at the engine face, a flow angle survey at the inlet throat and engine face, surface static pressure measurements along the forebody and inlet walls and laser light sheet flow visualization of the flow ahead of the inlet. The effects of mass flow rate, angle of attack and yaw will be investigated.

In addition to the model scale test program, a flight test program is also planned to be conducted at the NASA Dryden Flight Research Center using a specially equipped F/A-18 called the High Alpha Research Vehicle (HARV). This program will provide inlet duct data such as surface static pressures and a compressor face total pressure distribution. This full scale data will be compared with CFD predictions to be obtained at Lewis. 


\subsection{Numerical Modelling}

In this section the major features of the PARC3D and CFL3D codes are presented along with a discussion of the boundary conditions used with each code. A detailed discussion of the development of the computational grid is presented.

\subsection{PARC3D Code}

The PARC3D code [Ref 5] solves the full three-dimensional Reynolds averaged NavierStokes equations in strong conservation form using the Beam and Warming approximate factorization scheme to obtain a block tridiagonal system of equations. Pulliam's scalar pentadiagonal transformation provides for an efficient solver. The code uses the BaldwinLomax turbulence model [Ref. 9]. Specifically, the NASA Lewis version of this turbulence model does not use the streamwise component of vorticity in the determination of the turbulent viscosity [Ref. 10]. This approach was found to improve the modelling of vortical flows in ducts. The implicit scheme uses central differencing with artificial dissipation to eliminate oscillations in the solution associated with the use of central differences. This code allows for the use of multiple grid blocks. Trilinear interpolation [Ref. 11] is used to transfer information at the grid block interfaces.

\subsection{CFL3D Code}

The CFL3D code [Ref. 8] solves the thin-layer Navier-Stokes equations. It employs the Baldwin-Lomax [Ref. 9] turbulence model. The equations are solved using upwind differencing with a total variation diminishing (TVD) scheme. The TVD scheme eliminates oscillations due to dispersion errors introduced by the higher order terms in the upwind differences by shutting off these higher order terms in regions of large flow oscillations. Various options are available in the code for TVD schemes, flux-vector differencing, and upwinding accuracy. The options chosen were the Roe flux difference splitting scheme, third order accurate upwinding, and the smooth flux limiter which is tuned for the third order accurate upwinding scheme. The three-factor approximate factorization scheme is used to obtain a block tridiagonal system of equations. For the Roe scheme, the equations are diagonalized to obtain a scalar tridiagonal system of equations. This diagonalization provides for a more efficient solver. A semi-conservative scheme is employed to transfer information between grid blocks [Ref. 12]. Multigridding is also available which may accelerate the convergence of the solution [Ref. 13]. 
However the multi-gridding option could not be implemented for this problem. This was due to the fact that the grid block boundary interfaces require very close match of adjacent grid block edge boundaries which could not be maintained along complex interfaces as the grids were coarsened during the multigrid cycle. The use of multigridding has the potential to reduce the required computational time by up to a factor of three.

\subsection{Grid Generation}

Accurate modeling of the geometry and judicious clustering of grid points is needed for a correct numerical solution and an economical computation. The complex multi-block grid used for these calculations was created with the GRIDGEN grid generation system [Ref. 14]. This grid generator has proven to be very effective in creating complex multiblock grids.

The geometry database used to create the grid for this study was obtained from McDonnell-Douglas. The database consisted of $x, y, z$ coordinate points given at axial cuts along the fuselage. The database included the definition of the fuselage, LEX, wing, tail, ramp and the inlet. The original grid based on the initial database did not include or have sufficient definition of the diverter, the LEX slot, and the inlet highlight. The deficiencies in the geometry database used for this grid have been corrected and a revised grid, created with the more accurate database, was generated.

Due to the complex interactions between the fuselage flow field and the inlet flow field, the forebody, fuselage, LEX, ramp and wing were all included in the grid. The horizontal tail, vertical tail, aft fuselage and the nozzle were not modeled because of their minimal effects on the inlet flow field. The wing leading edge flap, which is deflected down 34 degrees when the aircraft is at 30 degrees angle of attack and greater, was modeled undeflected in the original grid. The deflected flap was included in the revised grid. An embedded C-grid about the inlet highlight is also included in the revised grid to improve the resolution of flow gradients and surface geometry. A pair of counter rotating vortex generators which are positioned approximately halfway downstream from the inlet entrance in the inlet duct were not modelled due to their small size, which would require an exorbitant number of grid points to resolve. Due to the CFL3D code not allowing for over-lapping grids, the inlet highlight c-grid and the wing lending edge flap were removed from the revised PARC3D grid for generating the CFL3D grid. 
The CFL3D code stores all data in core memory which requires significantly more space than PARC3D which keeps only the data for the block being analyzed in core memory. The rest of the block data is stored on disk memory, therefore the total number of grid points used for the CFL3D grid was less than the revised PARC3D grid due to core memory limitations.

The database was modified in selected areas, as discussed below, using the program I3G/VIRGO (Interactive Graphics for Geometry Generation, Ref.15). This program was developed at the Wright Research and Development Center, and it runs interactively on the IRIS workstation. It is mainly used for geometry database manipulation, such as rotations, translations, and scaling. In addition, it is used to reformat databases to the one compatible with the GRIDGEN system. It can also be used to join together, or split apart surface definitions. For instance, the top of the LEX was split from the bottom of the LEX in order to generate a viscous grid under the LEX and an inviscid grid above the LEX. I3G/VIRGO is also useful for geometry generation using lines, elliptic arcs, and curves. These features were used to enhance certain areas of the database such as the ramp and the wing root, which were not defined well in the initial database. In addition, the I3G/VIRGO features were used to create the definition of the external cowling for the original grid. Finally, the database was scaled to match the $19.78 \%$ scale wind tunnel forebody/inlet model which will be tested at NASA Lewis $9 \times 15$ wind tunnel.

The first program in the GRIDGEN grid generation system is GRIDBLOCK. This is an interactive program which runs on the Iris workstation. This program was used to create the blocking structure of the grid. The blocking structure was affected most significantly by the degree of complexity of the surface geometry. The blocking structure was also affected by the flow solver boundary condition requirements and block interface requirements. Another factor in determining the blocking structure was the maximum number of grid points per block allowed by computer memory limitations.

The blocking structure used for the F/A-18 consists mainly of C-grids wrapped around the fuselage, in the cross-flow plane. The original F/A-18 blocking structure can be seen in Figure 2a. The revised F/A-18 blocking structure is shown in Figure 2b. The inlet block is an O-grid extending from the inlet lip to the compressor face. The blocks were created such that the blocks under the LEX contained grids for viscous flows, while the blocks over 
the LEX were sparser for inviscid flow analysis. This was done because it was felt that the viscous effects above the LEX did not significantly effect the flow into the inlet. This also decreased the required number of grid points and thus the run times. Blocks were extended approximately one body length away from the surface in order to minimize the effects of the free stream boundary condition on the solution near the inlet.

Once the blocking structure was completed, the second GRIDGEN program, GRIDGEN2D, was used to create the grid on the six block faces. This program, like GRIDBLOCK, runs interactively on the Iris. GRIDGEN2D contains a variety of stretching functions for point distribution on face edges. The stretching function which was used in the majority of cases for this grid was the one based on Vinokour's functions (Ref 16). The viscous grids were packed so that spacing at the wall was $.0002 "$. This produced a $\mathrm{Y}+$ of around 1. After the edges on a face are defined the points on the interior of the face are defined. An algebraic solver using transfinite interpolation with arc based interpolants was used to initialize the grid for most faces. If the resulting grid needed smoothing or orthogonality along an edge, the elliptic solver was used. The Thomas and Middlecoff method (Ref 17) along with Sorenson's method (Ref 18) proved to be the most effective.

The third GRIDGEN program, GRIDGEN3D, is a batch code that runs on a Cray computer. It creates the grid in the interior of each block. Most of the blocks only required the algebraic solver to initialize the interior points. However, some blocks with excessive skewness required the elliptic solver to smooth the grid. GRIDGEN3D also creates a partial PARC3D input file based on the boundary conditions specified in GRIDBLOCK. The original grid contained approximately 1.1 million grid points and the revised grid contained approximately 1.2 million points.

The grid on the symmetry plane and on the F-18 surface can be seen in Figure 3a for the original grid and for the revised grid is shown in Figure 3b. A close-up view of the grid along the airframe surface is shown in Figure 4a for the original grid and the revised grid is shown in Figure $4 \mathrm{~b}$.

One of the main problems with creating a multi-block grid is maintaining correct interfaces were two blocks meet. Many multiblock CFD codes, such as CFL3D, require that all the face points of an interface must lie along the boundaries of the adjoining block. This requirement is impossible to meet with non-contiguous interfaces with curved edges 
using a linear interpolation scheme. This is most difficult when a grid is packed very closely, for a viscous wall boundary condition, at one of these edges. The only solution is to carefully distribute the finer grid points along the curved edge such that each point on the edge lies entirely along the other coarser grid block's boundary, in terms of the linear interpolation scheme used to capture all the points. For overlapping grid boundaries, as required by PARC3D, it may be difficult to contain the extended grid boundaries outside of solid surfaces at complex geometric boundaries.

\subsection{Boundary Conditions}

\subsubsection{PARC3D Code}

The farfield type boundary conditions were imposed along the outer computational boundaries. This type of condition uses a one-dimensional Riemann invariant to maintain the free-stream flow conditions. Along the windward side of the airframe, no-slip, adiabatic conditions are specified. Along the leeward side of the aircraft, inviscid flow is specified. The static pressure at the engine face was adjusted to obtain the equivalent experimental corrected mass flow rate based upon total pressure at the engine face plane obtained from an average of 40 total pressures in a configuration of 8 equally spaced legs with 5 equal area total pressure locations per leg. Symmetry conditions were assumed along the centerline of the aircraft. Trilinear interpolation is used to transfer data between the grid blocks.

\subsubsection{CFL3D Code}

The boundary conditions used by CFL3D were similar to the PARC3D conditions with a few exceptions. The CFL3D code does not have a turbulence model with generalized wall boundary conditions which allow the use of opposing walls within a single grid block. Therefore, the flow in the diverter, LEX slot and upper divertor was treated as laminar. The effects on the predicted flow field of using the laminar flow assumption are discussed in this report. Also, since the grid block interface boundary conditions are for a full block surface, the slot exit was modelled as a subsonic exit pressure boundary instead of an interface to the top side of the LEX since the slot interface made up only a portion of the adjoining block's surface. The exit static pressure was set based upon the average static pressure calculated by the PARC3D code which used an interface boundary condition. In order to model the slot interface with the CFL3D code, the use of several grid blocks on 
the top side of the LEX would be required to isolate the slot interface along a complete grid block surface. 


\subsection{Results}

In this section, the results using the original and revised grids with PARC3D and the CFL3D solution are presented. The external flow results are presented first and followed by a discussion of the inlet duct flow field predictions. The results are compared with available data.

\subsection{External Flow Field}

In this section, the external flow features common to all three solutions will be discussed first. Following this, the differences in the three external flow results will be presented. The graphical results presented in this section were obtained using the PLOT3D program [Ref. 19].

At 30 degrees angle of attack, a vortex is formed underneath the LEX as can be seen in Figure 5. The predicted particle traces (simulated oil flows) along the forebody and along the fuselage below the LEX are shown in Figure 6. The windward vortex is generated when the flow impinges on the bottom of the LEX and moves down the fuselage until it reaches a separation line. The PARC3D solution using the revised grid indicates that the under LEX vortex has moved downward along the fuselage as compared to the flow pattern shown for the original grid. The discontinuity in the particle traces shown in the original grid is due to the use of two blocks, instead of one as in the revised grid. The PLOT3D code will not allow particles to pass between two grid blocks when the particles are restricted to a surface, unless the two surfaces are coincident. The increased curvature in the particle traces using the revised grid may be due to the presence of the slot through which flow is passing which causes an upward movement of the traces near the entrance to the diverter region. In addition, the presence of the flow splitting "wedge" may be providing blockage to the flow.

The particle traces obtained using the CFL3D solution with the revised grid are somewhat different than the particle traces predicted by PARC3D using the revised grid. These discrepancies between the PARC3D and CFL3D results may be due to the fact that the revised grid used for the CFL3D calculations along the lower LEX region of the fuselage had approximately half the number of streamwise points as the revised PARC3D grid due to the core memory storage limitations of CFL3D. In addition, the version of the BaldwinLomax turbulence model used in the PARC3D solutions included only the component of 
vorticity which is normal to the streamwise direction in the determination of the turbulent viscosity whereas the CFL3D code uses the total vorticity. This difference can affect the position of the vortex as discussed in Ref. 10. Another possible source of the discrepancies could be due to the different algorithms used. The PARC3D code uses central differencing with artificial dissipation while the CFL3D code using a third order upwinded differencing with a TVD scheme. The amounts of numerical dissipation introduced into the solution by these two schemes could be quite different.

Another effect on the vortex trajectory may be due to the modelling of the flow behind the ramp as is shown in the close-up view of the particle trajectories slightly ahead of the ramp in Figure 7. A comparison to the PARC3D results with the original and revised grids shows that the inclusion of the diverter and slot in the revised grid causes the flow to move slightly upstream from the ramp and upwards through the slot. The flow behind the ramp in the original grid was modelled inviscidly, without a slot or diverter, which resulted in the flow moving parallel to the axial direction. The CFL3D results show the flow moving farther upstream than the PARC3D results using the revised grid. This discrepancy in the two solutions may be attributed to the use of the laminar flow approximation in the CFL3D solution in the diverter/slot grid blocks. The laminar flow tends to have larger regions of cross-flow separation than the turbulent case, and may affect the flow directionality. Since the CFL3D code turbulence model does not allow the use of opposing walls within a single grid block, the use of several more grid blocks would have been necessary to model the diverter/LEX slot regions.

The effects of the improved modelling of the upper diverter (see Fig. 1b) on the flow along the ramp are shown in Figure 8. The particle trajectories obtained for the original grid indicate that there is a significant region of separated flow along the ramp near the top of the inlet. This may be due to the lack of adequate geometry resolution of the upper diverter region in the original grid. This region was modelled as an inclined flat plate due to this lack of definition in the original data base. In the revised grid, this region is accurately defined in the data base and much more aerodynamically contoured to allow flow to move over the top of the inlet and results in small amounts of upstream movement of the ramp particle traces in the revised grid results. The CFL3D results indicate that the flow has a saddle point upstream, although the particle trajectories appear to enter 
the inlet at a location similar to that in the revised PARC3D results. This saddle point may be due to modelling the cove as a laminar flow region which has a different crossstream separation pattern than the turbulent case in PARC3D. This region was modelled as laminar due to the presence of opposing wall in a single grid block which cannot be handled by the present turbulence model.

Surface static pressure measurements from a flight test were made at several fuselage locations as shown in Figure 9. A previous computational study for fully turbulent flow [Ref. 4] indicated that the effects of $19.78 \%$ scale were negligible for this type of data and therefore full scale data could be compared with these subscale results. The numerical results for the surface static pressures along the forebody and LEX are all in good agreement with the data [Ref. 6], as shown in Figure 10, despite differences in the vortex location. The CFL3D results appear to have the closest agreement with the data, although the other solutions are almost as close. The discrepancies between the numerical results and the data along the forebody may be due to the lack of adequate grid resolution of the vortex dominated region of the flow. Along the top side of the LEX the agreement is poor due to lack of grid resolution and the use of the inviscid flow approximation in this region. Another source for the discrepancies may be due to laminar to turbulent transitioning flow which is present on the full scale vehicle [Ref. 6]. These calculations assume fully turbulent flow present everywhere.

\subsection{Inlet Duct Flow Field}

In this section, several aspects of the inlet duct flow field are examined. Comparisons of the predicted surface static pressure distributions about the inlet highlight are first presented. This is followed by a discussion of the predicted total pressure contours at the inlet entrance and engine face. The predicted engine face total pressures contours are compared with experimental total pressure contours. The predicted inlet performance is also compared with data. The vorticity contours depicting the secondary flow field and vortices present in the three solutions are then presented for the duct entrance and engine face stations.

The surface static pressure distributions about the inlet highlight obtained from the three solutions are shown in Figure 11. As can be seen the distribution obtained using the embedded c-grid is much improved over the original solution. It should be noted 
that the pressure distribution obtained with the original grid without the c-grid does not adequately resolve the supersonic acceleration about the highlight and there is a significant total pressure overshoot (10\% greater than freestream).

The CFL3D pressure distributions compare much closer with those obtained by PARC3D using an embedded c-grid about the inlet highlight than the original grid results. The poor pressure distributions obtained with PARC3D without a c-grid about the leading edge highlight may be due to the presence of a discontinuity, in the grid distribution in the axial direction, at the inlet entrance grid block interface which extends into the upstream flow field. Due to the complex geometry, manually extending the grid interface to maintain continuous metrics is difficult. Also, the number of grid points in the region of the highlight for the original grid is approximately one-half the number used in the CFL3D grid.

For the CFL3D code, the grid block surfaces do not have to be overlapped. The CFL3D code uses an approach that is similar to the PARC3D in regards to transferring information between grid blocks. Essentially, CFL3D creates two layers of ghost cells within the adjacent block and assumes that the grid spacing normal to the interface boundary is approximately the same on both sides of the boundary. If the normal spacing is not the same, errors similar to those discussed for the overlapping boundary used for PARC3D will be encountered. These ghost cells are used to interpolate data from the adjacent grid block. For PARC3D, at least one additional overlapping surface must be generated in order to interpolate data from the adjoining grid block. With PARC3D, interpolation coefficients for all three directions are determined by the location of the interface surface. Whereas, CFL3D assumes the grid spacing normal to the block interface is the same on each side of the block interface and only determines the interpolation coefficients in the tangential directions to the interface surface using the actual coordinates of the grid points defining the block interface surface.

The predicted total pressure contours at the inlet entrance obtained for each solution are shown in Figure 12. The boundary layers are significantly thinner for the revised grid results as compared to the original grid solution. This improvement is due to the c-grid providing better resolution of the flow gradients about the lip highlight. These gradients are severe since the stagnation point is located along the outside of the inlet lip region and 
flow must be turned 180 degrees as it enters the inlet. The total pressure contours obtained from the CFL3D results indicate a slightly thicker boundary layer than that obtained with PARC3D using the embedded c-grid. However, the losses associated with the CFL3D solution appear to be somewhat lower than the PARC3D results obtained without the c-grid. A region of cross-flow separation is shown in the revised grid and CFL3D grid solutions. The original grid does not indicate such a region. The improved resolution of the inlet highlight region appears to be the reason this feature is resolved in the revised grid and CFL3D grid results.

The predicted total pressure contours at the engine face, shown in Figure 13. The PARC3D results obtained with the revised grid are much less distorted than those obtained with the original grid. The major difference is the presence of the embedded c-grid about the inlet highlight in the revised grid which has a significantly higher grid density in the highlight region. This c-grid provided much lower losses at the inflow to the inlet and therefore the losses and distortions are reduced at the outflow station. A comparison of the predicted inlet performance using both grids with PARC3D to data [Ref. 7] is shown in Table 1. As can be seen, the results obtained using the revised grid indicate improved inlet performance over the original grid and much closer agreement to the data than the original grid results. It should be noted that the mass flow rate for the revised grid was set at two values: full scale $150 \mathrm{lbm} / \mathrm{sec}$ and $144.7 \mathrm{lbm} / \mathrm{sec}$ which is the present design flow rate for this inlet. The reduction in flow rate resulted in an increase in inlet performance.

The PARC3D predicted distortions are less using the revised grid than the CFL3D predictions. Part of these distortions may be attributed to the lack of a C-grid around the inlet highlight for the CFL3D calculations. The use of the C-grid improved the PARC3D calculations significantly. However, even without the C-grid about the highlight, the total pressure contours near the inlet entrance predicted with CFL3D indicated only a slightly thicker boundary layer than the PARC3D results obtained with the revised grid. In addition, the CFL3D surface static pressure distribution about the highlight is much closer to the $\mathrm{C}$-grid results than the original grid results. The pole boundary condition used in the CFL3D computations is the most significant source of the distortions at the engine face. The pole influences the CFL3D solution by distorting the flow field in the vicinity of the pole. This behavior of the CFL3D pole boundary condition in the presence of flow 
gradients has been observed in computational results concerning the flow in diffusing sducts, the cause of which has not been determined. The effects of the pole in the PARC3D solution are much less significant. In Table 1, a comparison of the CFL3D predicted inlet performance to the PARC3D revised grid results shows that the CFL3D predictions have a lower recovery and significantly higher distortion than the PARC3D results. The PARC3D performance predictions compare closer to the data.

A comparison of the PARC3D normalized total pressure contours using the revised grid are compared to data obtained with a $19.2 \%$ scale model for the F-18 aircraft [Ref. 7] in Figure 14. The normalized total pressure represents the percent deviation from the rake averaged total pressure at the engine face. In Figure 14a, the measured time-averaged normalized total pressure is shown and the measured normalized total pressure at peak distortion is shown in Figure 14b. As can be seen, there is a significant difference between the two experimental results. This indicates a large amount of unsteadiness may be present in the inlet duct flow field. The PARC3D results appear much more similar to the peak distortion data than the time-averaged experimental result. It should be noted that the solutions obtained are converged to an asymptotic, non-time varying condition and not time accurate therefore, the solutions obtained cannot duplicate the time-averaged data shown or the peak distortion data. In order to attempt to match the time-averaged data, a time accurate solution is needed with the results at each time step averaged. This issue also raises the question of unsteady turbulence modelling capability.

The predicted vorticity contours inside the inlet entrance are shown in Figure 15. The position of the ingested vortex in the original grid results is close to the inboard wall. The vortex position in the PARC3D and CFL3D solutions using the revised grid is towards the top center of the inlet entrance. In the case of the original grid the vortex migrates towards the center of the engine face. For the revised grid cases, the vortex moves towards the inboard wall and dissipates in the wall boundary layer. This migration into the inboard wall may be due to the improved upper cowl definition which allows more of the external flow to move around the cowl instead of into it as shown in Figure 8.

The revised grid solutions using PARC3D and CFL3D indicate a region of crossstream separated flow along the lip shown in Figure 12. For the revised grid and CFL3D grid results this region is located along the outboard wall of the lip and migrates towards 
grid results this region is located along the outboard wall of the lip and migrates towards the bottom of the duct and merges with vortices which have migrated from the inboard corners of the duct. For the original grid results, this separated region is not present. This difference is attributed to the increased grid resolution in the revised grid which utilizes an embedded C-grid around the inlet highlight. These vortices appear to be major contributors to the development of the inlet duct secondary flow field. This indicates that the effect of the lip does have a significant impact on the development of the secondary flow field in inlets. This migration was exhibited by using the animation capabilities of the FAST program [Ref. 20]. It should be noted that these vortices contain very low energy flow and appear to be a significant contributor to the high levels of distortion that have been predicted at the engine face. Since data indicates that these distortions at the engine face levels are too high, these vortices may not be real or not as intense and may be attributed to a lack of adequate inlet highlight resolution. The calculated vorticity contour plots of the flow field at the engine face are shown in Figure 16. The PARC3D vorticity plot using the original grid indicates a large vortex along the bottom of the duct which is due to the development of secondary flow in the duct. A second vortex near the center of the duct is also present. This vortex is the under LEX vortex that has been ingested. This vortex accounts for the large migration of low energy flow about the center of the duct exit shown in Figure 13a. The PARC3D solution using the revised grid indicates a single vortex at the compressor face due to secondary flow development in the inlet duct. In the revised grid the LEX vortex is also ingested but migrates into the inboard duct wall as discussed previously.

The CFL3D vorticity contour plot at the engine face indicates several small vortices present. The predicted flow field has not reached a numerically converged state with the number and location of these vortices changing as the solution is iterated. Although the PARC3D solution exhibited some non-steady state convergence, the general features at the engine face remained fairly constant with a minimum of changes. The discrepancy in the CFL3D solution may be attributed to the pole boundary condition which introduces significant distortions in the vortical shear layer as it moves into the pole region of the grid. Another possible reason for the lack of steady-state convergence may be that the CFL3D solution contains less numerical dissipation than the PARC3D solution. 


\subsection{Numerical Issues}

A comparison of the numerical performance of the two codes, shown n Table 2, indicates that CFL3D is faster but requires significantly more in-core memory than PARC3D. This is due to the fact that CFL3D stores all of the numerical data in core memory, whereas PARC3D keeps only the current grid block data in core memory and writes the rest to disk memory. Although difficult to assess, both codes required approximately the same amount of computational time to obtain a converged solution. Some differences in CPU time may be attributed to PARC3D requiring much more I/O than CFL3D since data for only one grid block is stored in core memory using the PARC3D code. The drop in the residuals for both codes was similar. The residuals for the viscous blocks dropped approximately three orders of magnitude while the inviscid blocks dropped up to six orders of magnitude.

The calculations of lift and drag using the revised grid for PARC3D and CFL3D as a function of iterations indicate the CFL3D solution has less steady state convergence than the PARC3D solution. The lift and drag coefficients calculated for PARC3D were virtually constant. The CFL3D lift coefficient varied by $+/-3.5 \%$ and the drag coefficient varied by $+/-1.5 \%$. The major source of this lack of convergence is the leeward side of the wing. A difference in the PARC3D and CFL3D flow field solution convergence was found also to be the case in the inlet duct flow field calculation. The CFL3D predicted mass flow rate varied $+/-1 \%$ while the PARC3D calculated mass flow rate variation was $+/-.4 \%$ as the solution was iterated. The non-convergence of the inlet flow field in the CFL3D solution may be attributable to the pole boundary condition influencing the flow field in this region. However, since there is a large disparity between the measured time-averaged compressor exit total pressure contours and those measured at peak distortion, the inlet duct flow field may be inherently unsteady and the CFL3D solution is indicating this in the results. To pursue this issue further, the pole boundary condition problem would have to be resolved. 


\subsection{Conclusions and Recommendations}

The grid generation system, GRIDGEN3D, performed reasonably well in the development of the F/A-18 computational grid. The additional components and refined geometry definition had a significant impact on the numerical results.

The PARC3D forebody/fuselage surface static pressures compared well with the flight test data and showed an improvement over the original grid results. The inclusion of the diverter and slot in the revised grid solution had an effect on the path followed by the under LEX vortex. The forebody/LEX surface static pressures obtained with CFL3D compared well to the PARC3D results and with data. However, the predicted vortex trajectory was different. This discrepancy may be due to the fact that the CFL3D solution used approximately one-half the number of streamwise points than PARC3D and differences in the turbulence models. Despite these discrepancies in the PARC3D and CFL3D solutions, the position of the ingested vortex within the inlet was very similar for both solutions. Therefore, the use of grid block splitting for each block which contained opposing walls in the CFL3D grid in order to model each wall with the minimum index to accommodate the present turbulence model was deemed unnecessary. However, in order to improve the CFL3D comparisons with PARC3D, the use of block splitting will be needed to model all viscous flow regions as turbulent. The problems with the use of opposing walls in the CFL3D turbulence model could be resolved by modifying the turbulence model to accept opposing walls and eliminate the need for grid block splitting.

Also, the refined definition of the upper divertor region above the inlet cowl appears to have had a significant influence on the migration of the ingested vortex. The original grid indicates that the vortex migrates to the compressor face while the revised grid indicates that the vortex dissipates along the inboard wall of the inlet duct. The PARC3D inlet flow field calculations, using the revised grid, show a significant improvement in the recovery and distortion over the original grid. This improvement appears to be due to the improved modelling of the inlet highlight and upper divertor region. However, PARC3D still overpredicts the inlet flow distortion and underpredicts the inlet total pressure recovery. Further refinements to the highlight grid and the inclusion of vortex generators may reduce the distortion. The possible unsteadiness in the inlet flow field may require the use of a time accurate solution. The PARC3D code predicted the inlet flow field better 
than CFL3D when compared to data. The major source of this discrepancy appears to be the CFL3D pole boundary condition introducing distortions into the flow field. One approach to correcting this problem would be to include an H-grid block along the inlet duct centerline and thereby eliminating the pole in the grid. Another contributing factor may be the lack of an embedded C-grid about the inlet highlight in the CFL3D simulation.

The effects of the inlet lip appear to have a significant effect on the development of the inlet duct secondary flow field. One approach to further verify this phenomena is to study the secondary flow development in the inlet duct using uniform inflow conditions. The difference in this secondary flow field and the one using the distorted inflow would be an indication of the contribution made by the inlet lip to the secondary flow field development. The vortices generated along the inlet highlight may be either fictitious or less intense and may be due to a lack of adequate grid resolution. The use of a more refined grid in this region would indicate the effects of grid density on the development of these highlight vortices. In order to compare numerical results with flight data, a fan simulation should be incorporated as the inlet exit boundary condition.

The PARC3D and CFL3D codes required approximately the same amount of computational time to obtain a converged solution. The PARC3D inlet mass flow rate was virtually constant while the CFL3D solution exhibited a $+/-1 \%$ mass flow rate variation in the duct. This non-convergence is partially attributed to the pole boundary condition introducing numerical disturbances into the flow field in regions where the vortical shear layer approaches the pole region. The CFL3D results may also be indicating the inherent unsteadiness of the inlet flow field which is indicated by experimental data.

One approach to correcting the multigridding problems would be to use all the fine grid points along a grid block boundary to define the coarser grid block boundaries. This would eliminate noncoincident boundaries developing at the coarser grid levels. 


\subsection{Acknowledgements}

Support of this work by the NASA Lewis Research Center of the National Aeronautics and Space Administration and under contract NAS3-25266 is gratefully acknowledged. The interest shown by Project Manager, Richard R. Burley, is particularly appreciated. The excellent grid generation work provided by James E. Bruns is also noted. In addition, the authors thank James $R$. Sirbaugh for his suggestions concerning multigridding with CFL3D and Raymond R. Cosner of McDonnell-Douglas Company for his help in obtaining the F/A-18 airframe geometry database. Also, the authors acknowledge the excellent work done by Maryann Johnston in preparing the text and figures for this report. 


\subsection{References}

1. Aeropropulsion '91, NASA CP 10063, Proceeding of a conference held at NASA Lewis Research Center, Cleveland, Ohio, March 20-12, 1991.

2. Ghaffari, F., Bates, B.L., Luckring, J.M., Thomas, J.L., and Biedron, R.T., "NavierStokes Solutions About the F/A-18 Wing-LEX-Fuselage Configuration with MultiBlock Structured Grids",AIAA Paper No. 91-3291, Presented at the 9th Applied Aerodynamics Conference, Baltimore, Maryland, September 23-25, 1991.

3. Gee, K., Tavella, D., and Schiff, L.B., "Computational Optimization of a Pneumatic Fuselage Forebody Flow Control Concept", AIAA Paper No. 91-3249, Presented at the 9th Applied Aerodynamics Conference, Baltimore, Maryland, September 23-25, 1991.

4. Bruns, J.E. and Smith, C.F., "Full Navier-Stokes Calculations on the Installed F/A18 Inlet at a High Angle of Attack", AIAA Paper No. 92-3175, Presented at the AIAA/SAE/ASME/ASEE 28th Joint Propulsion Conference and Exhibit, Nashville, Tennessee, July 6-8, 1992.

5. Cooper, G.K. and Sirbaugh, J., "The PARC Distinction: A Practical Flow Solver", AIAA Paper No. 90-2002, July 1990.

6. Fisher, D. F., Banks, D. W., Richwine, D. M., "F-18 High Alpha Research Vehicle Surface Pressures: Initial In-Flight Results and Correlation with Flow Visualization and Wind-Tunnel Data," NASA TM 101724, 1990

7. Amin, N.F., Hollweger, D.J., Franks, W.J., de la Vega, E.G., Yamada, M., and Tsukahira, T.W., "AEDC Series 1 F-18, .192 Scale Inlet Analysis Report", NOR 77-310, Northrop Corporation, Aircraft Division, Hawthorne, California, May 1977.

8. Thomas, J.L., Taylor, S.L., and Anderson, W.K., "Navier-Stokes Computations of Vortical Flows Over Low Aspect Ratio Wings", AIAA Paper No. 87-0207, Jan. 1987.

9. Baldwin. B.S. and Lomax, H., "Thin Layer Approximation and Algebraic Turbulence Model for Separated Turbulent Flows", AIAA Paper No. 78-257, Jan. 1978.

10. Sirbaugh, J.R., and Reichart, B.A., "Computation of a Circular-to-Rectangular Transition Duct Flow Field", AIAA Paper No. 91-1741, Presented at the 22nd Fluid Dynamics, Plasma Dynamics and Lasers Conference, June 24-26, 1991, Honolulu, Hawaii.

11. Stokes, M.L. and Kneile, K.L., "A Search/Interpolation Algorithm for CFD Analysis", 
Presented at the World Congress on Computational Mechanics, University of Texas, Austin, Texas, September 1986.

12. Bierdron, R.T. and Thomas, J.L., "A Generalized Patched-Grid Algorithm with Application to the F-18 Forebody with Actuated Control Strake", Computing Systems in Engineering, Vol. 1, Nos.2-4, pp. 563-576, 1990.

13. Anderson, W.K., "Implicit Multigrid Algorithms for the Three-Dimensional Flux Split Euler Equations", Ph.D. Dissertation, Mississippi State University, August 1986.

14. Steinbrenner, J. P., Chawner, J. R., and Fouts, C. L., "The Gridgen 3-D Multiple Block Grid Generation System," WRDC-TR-90-30222, 1991

15. "I3G/VIRGO: Interactive Graphics for Geometry Definition/Visual Interactive Rapis Grid Generation, User's Manual", Wright-Patterson AFB, January 1990.

16. Vinokur, M., "On One-Dimensional Stretching Functions for Finite-Difference Calculations," NASA CR-3313, 1980

17. Thomas, P.D., and Middlecoff, J.F., "Direct Control of the Grid Point Distribution in Meshes Generated by Elliptic Equations," AIAA Journal, Vol 18, 1979, pp. 652-656

18. Sorenson, R.L., "The 3DGRAPE Book: Theory, Users' Manual, Examples," NASA TM-102224, NASA Ames Research Center, July, 1989

19. Walatka, P.P., Buning, P.G., Pierce, L. and Elson, P.A., "PLOT3D User's Guide", NASA TM 101067, March 1990.

20. Walatka, P.P., Plessel, T., McCabe, R.K., Clucas, J., Elson, P.A., "FAST User's Manual", NASA Ames Research Center: WAO and RND, Beta 2.0, RND-91-011, December 1991. 


\begin{tabular}{|c|c|c|c|c|c|}
\hline & Orig. Grid & Rev. Gr & & CFL3D & DATA \\
\hline Full Scale Corrected Flow Rate: & 146.7 & 150.0 & 144.7 & 142.6 & 143.5 \\
\hline Model Scale Recovery : & $87.9 \%$ & $91.2 \%$ & $91.9 \%$ & $90.1 \%$ & $94.5 \%$ \\
\hline Model Scale Distortion: & $31 \%$ & $26 \%$ & $24 \%$ & $30 \%$ & $12 \%$ \\
\hline Engine Face Mach Number: & .46 & .48 & .45 & .45 & .45 \\
\hline
\end{tabular}

CFL3D PARC3D

Grid Points : $\quad 911,204 \quad 1,214,207$

Speed $(10 \mathrm{e}-6 \mathrm{~s} / \mathrm{it} / \mathrm{pt}): \quad 18.2, \quad 27.3$

Core Memory(MW)[1] : $\quad 28.9 \quad 6.5$

$\begin{array}{lll}\text { Words/Point : } & 31.7 & 32.4\end{array}$

1. For PARC3D, this is the memory required for the largest grid block.

Table 2. Code Performance Summary 


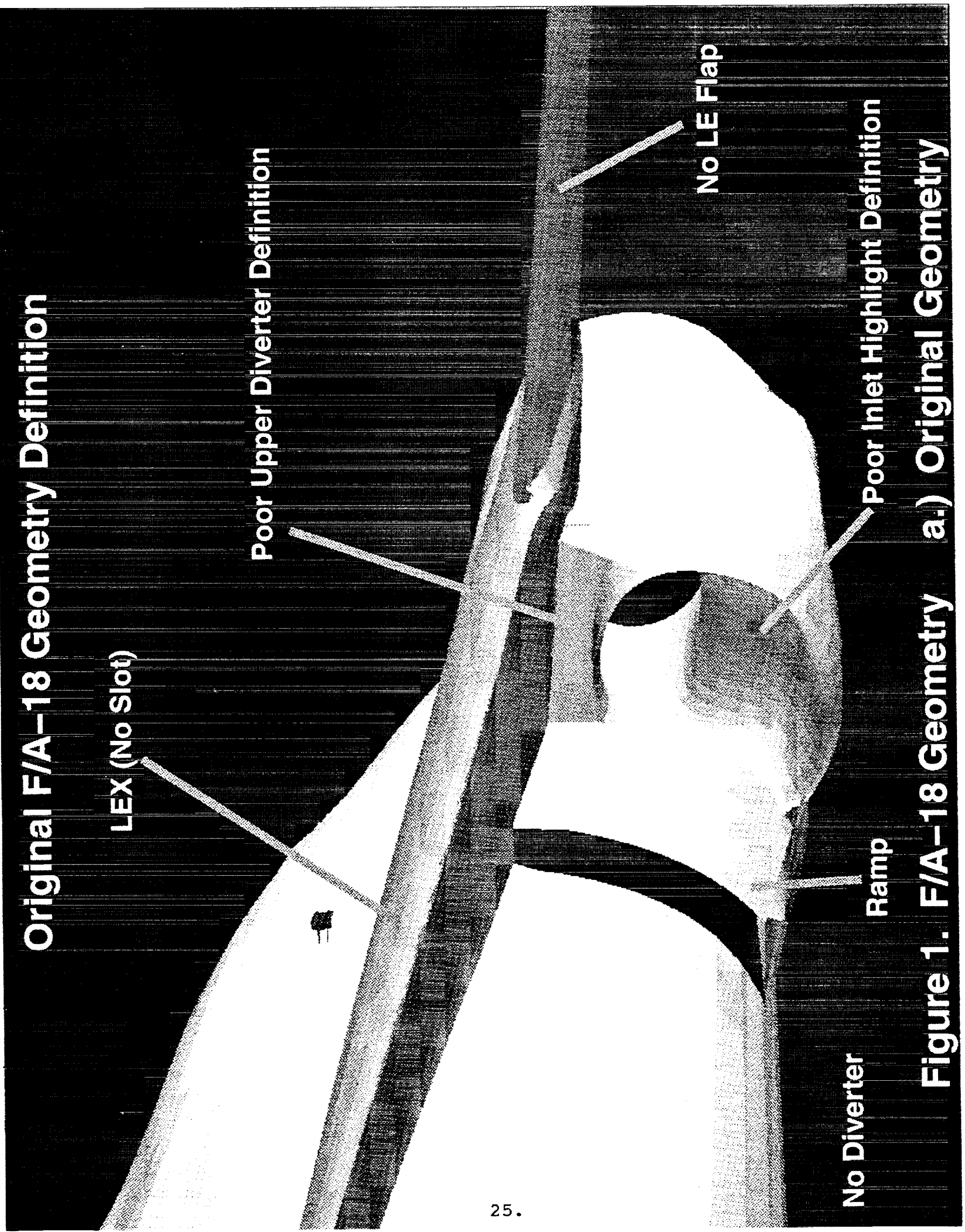




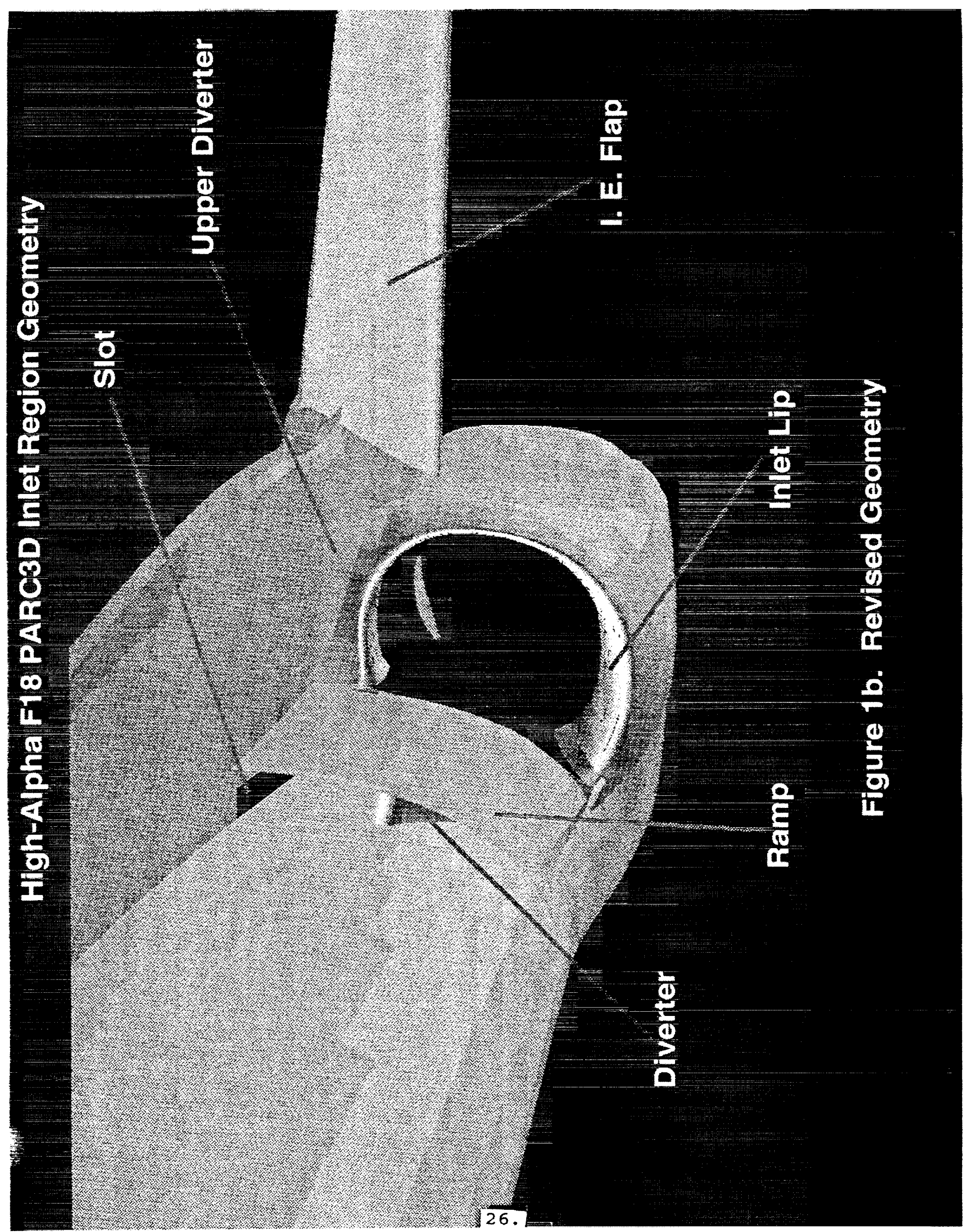




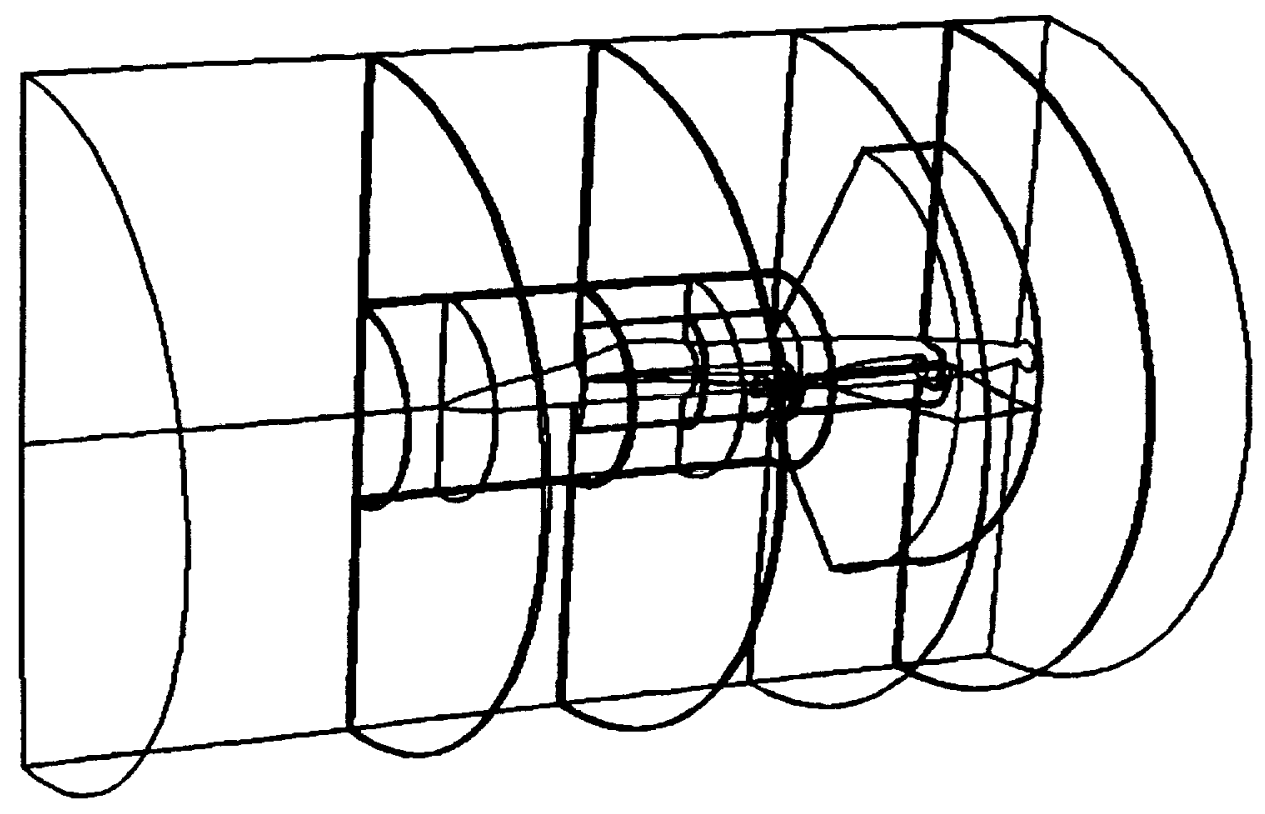

Figure 2a. Original Grid

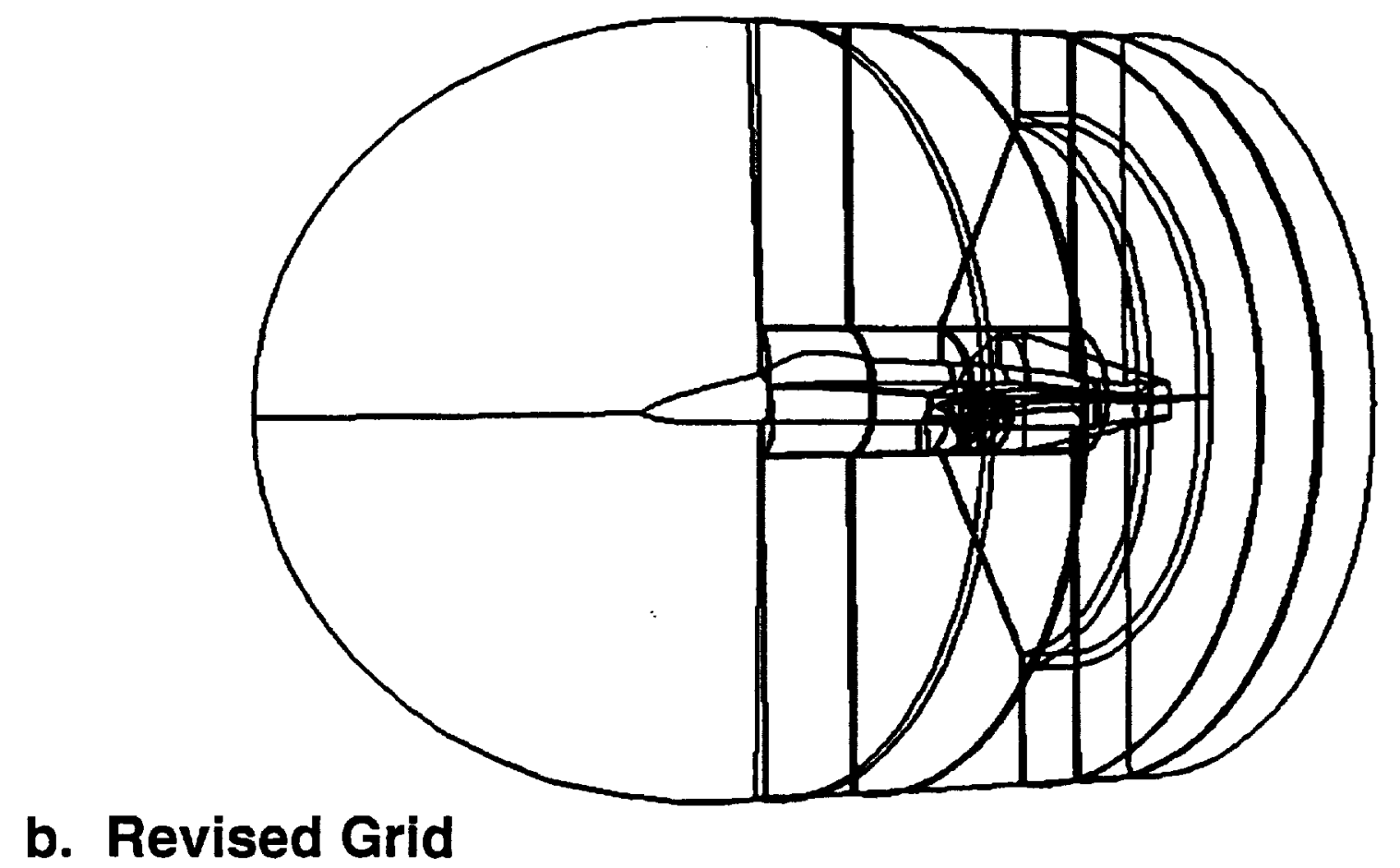

Figure 2. Grid Block Structure 


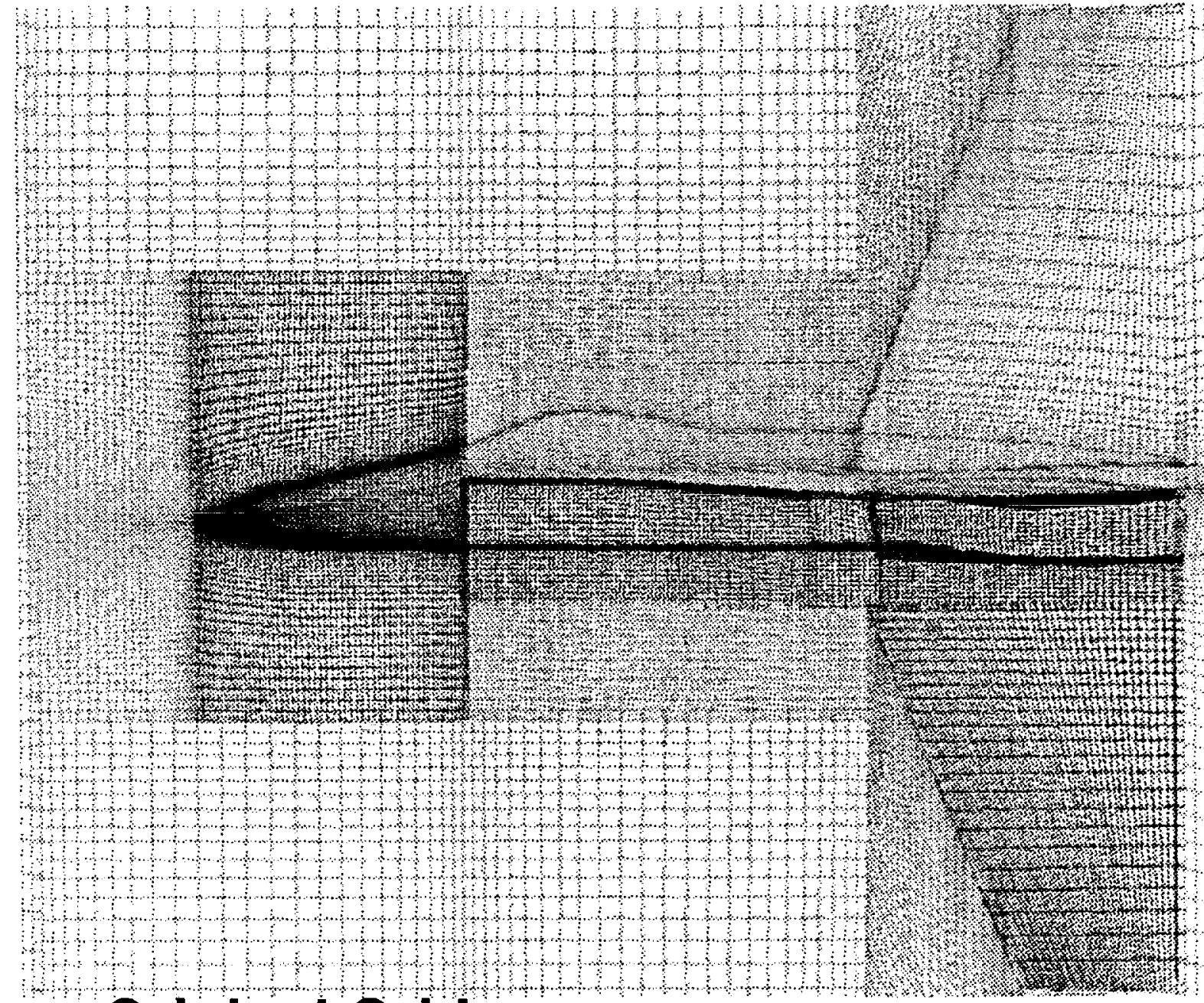

\section{a. Original Grid}

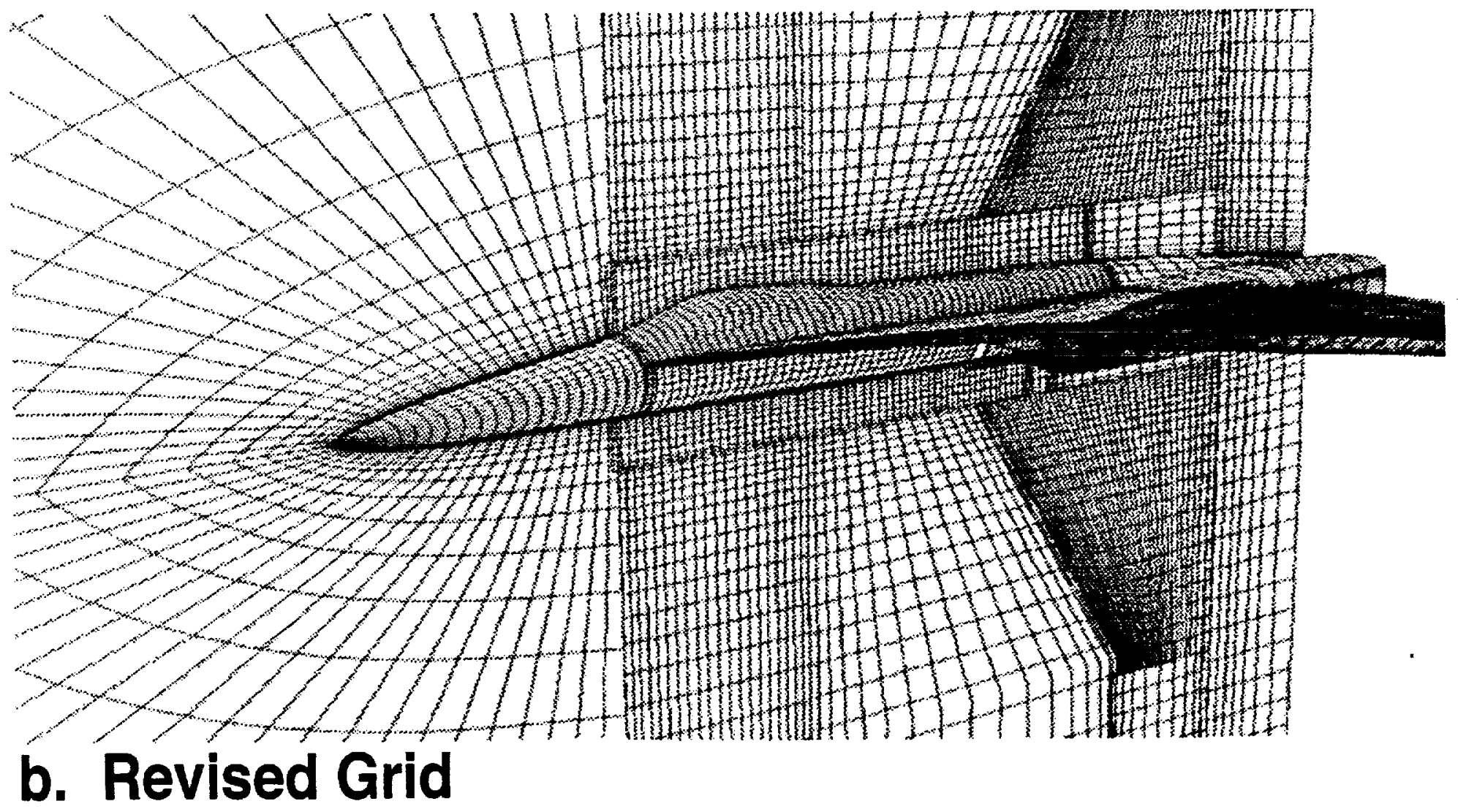

Figure 3. Plane-of-Symmetry View 


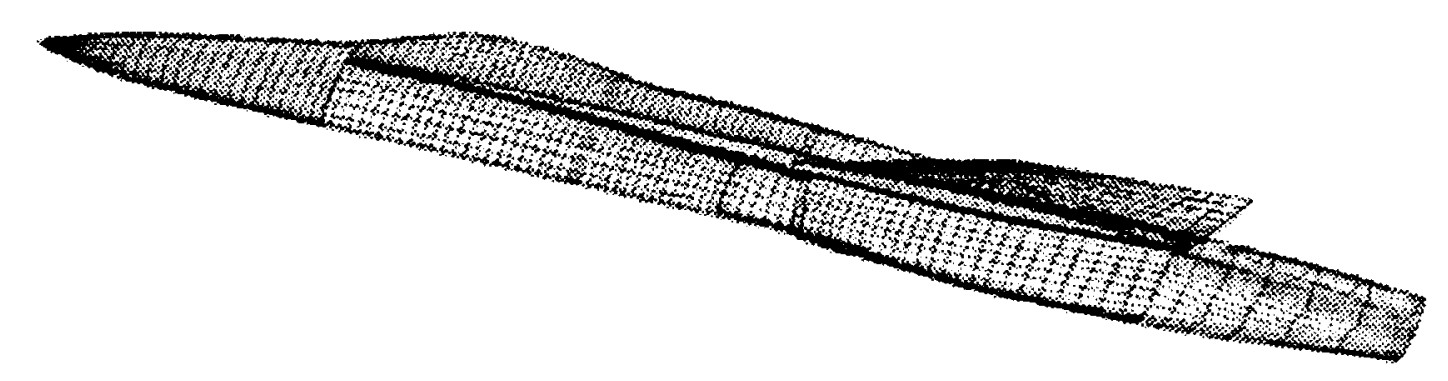

\section{a. Original Grid}

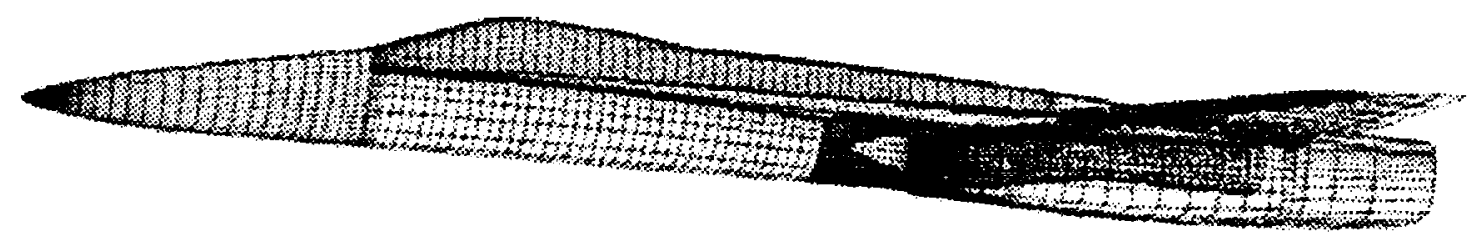

b. Revised Grid

Figure 4. Surface Grid (One-half points shown) 


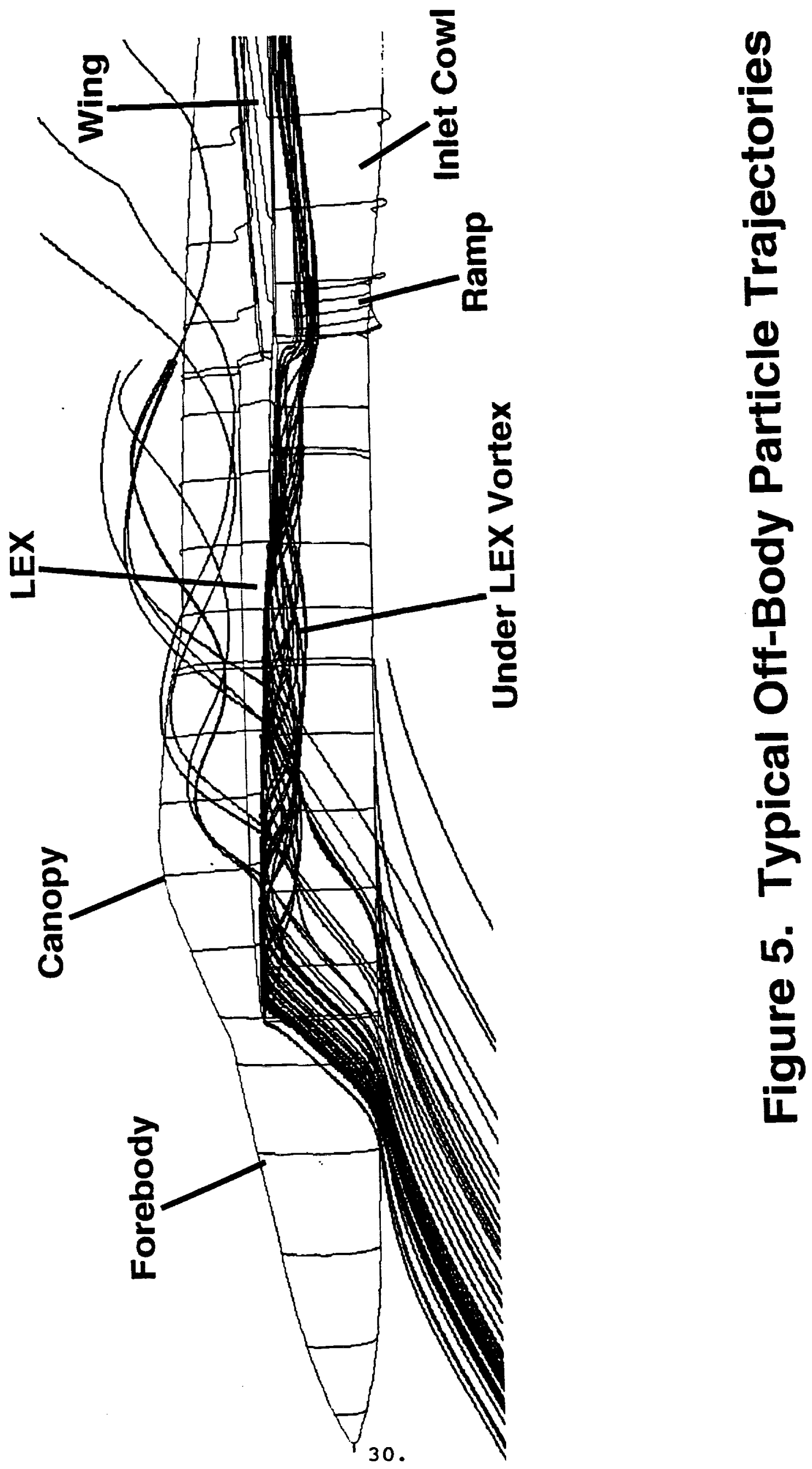




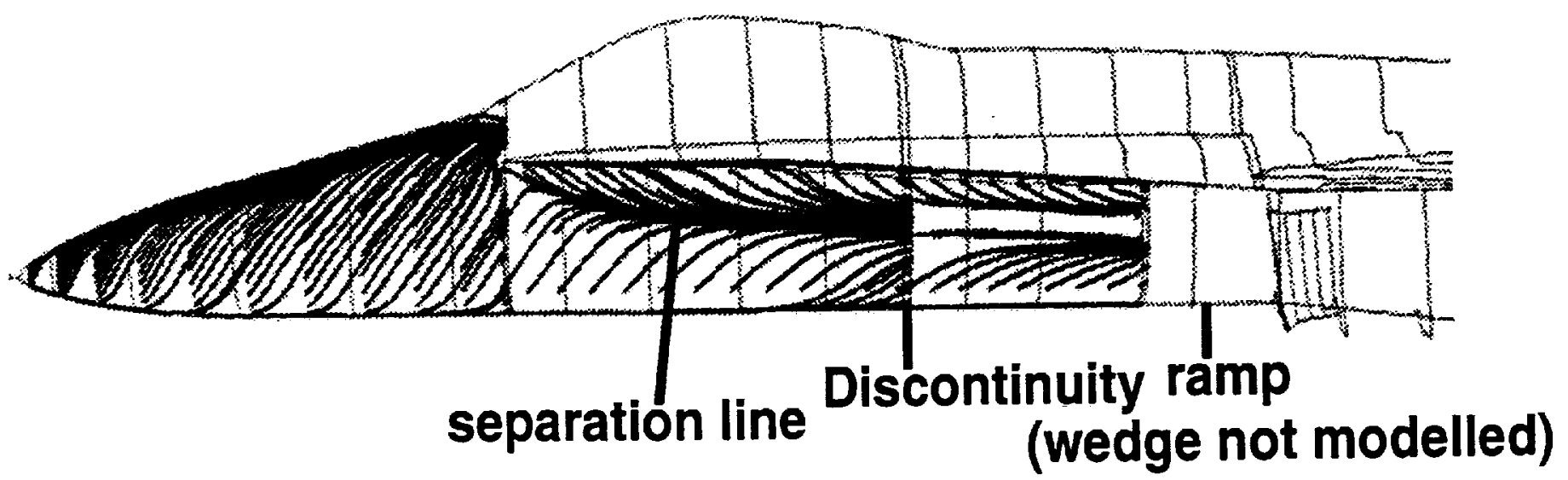

a. PARC3D Solution: Original Grid

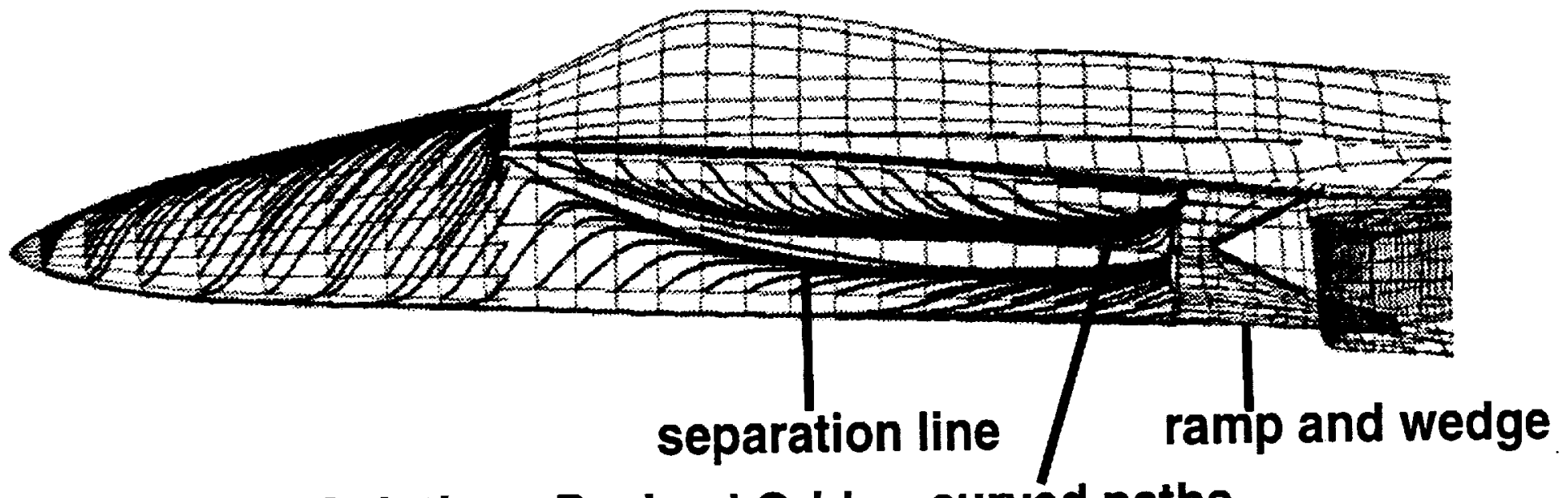

b. PARC3D Solution: Revised Grid curved paths

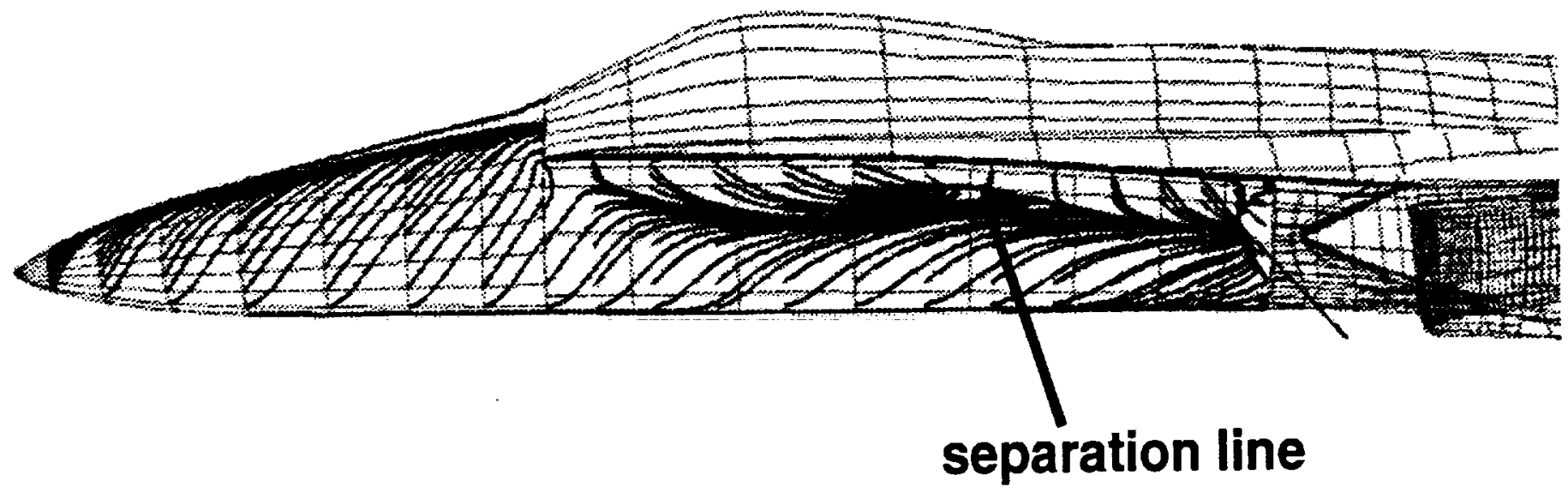

c. CFL3D Solution: CFL3D Grid

Figure 6. Particle Trajectories Along Surface 


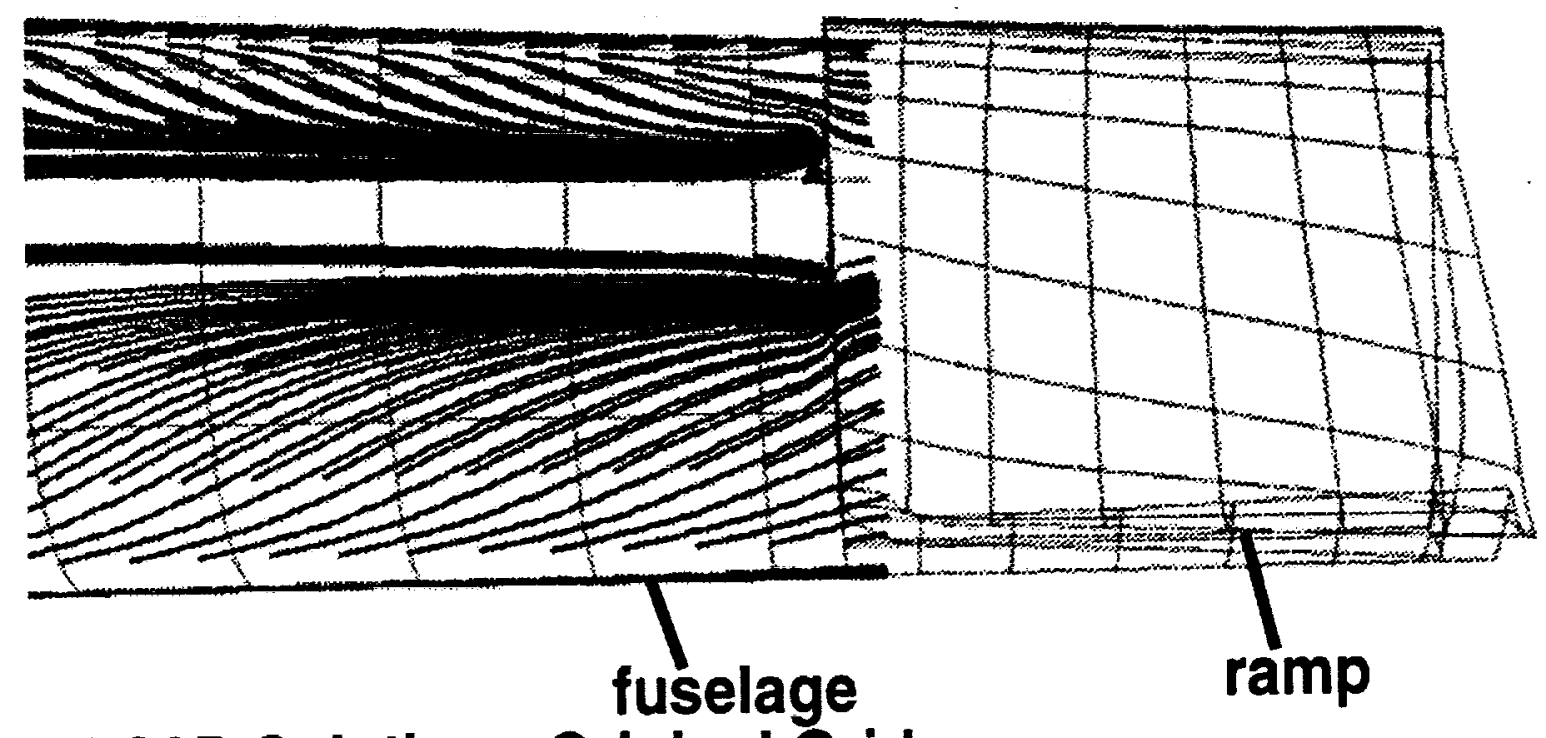

\section{a. PARC3D Solution: Original Grid}
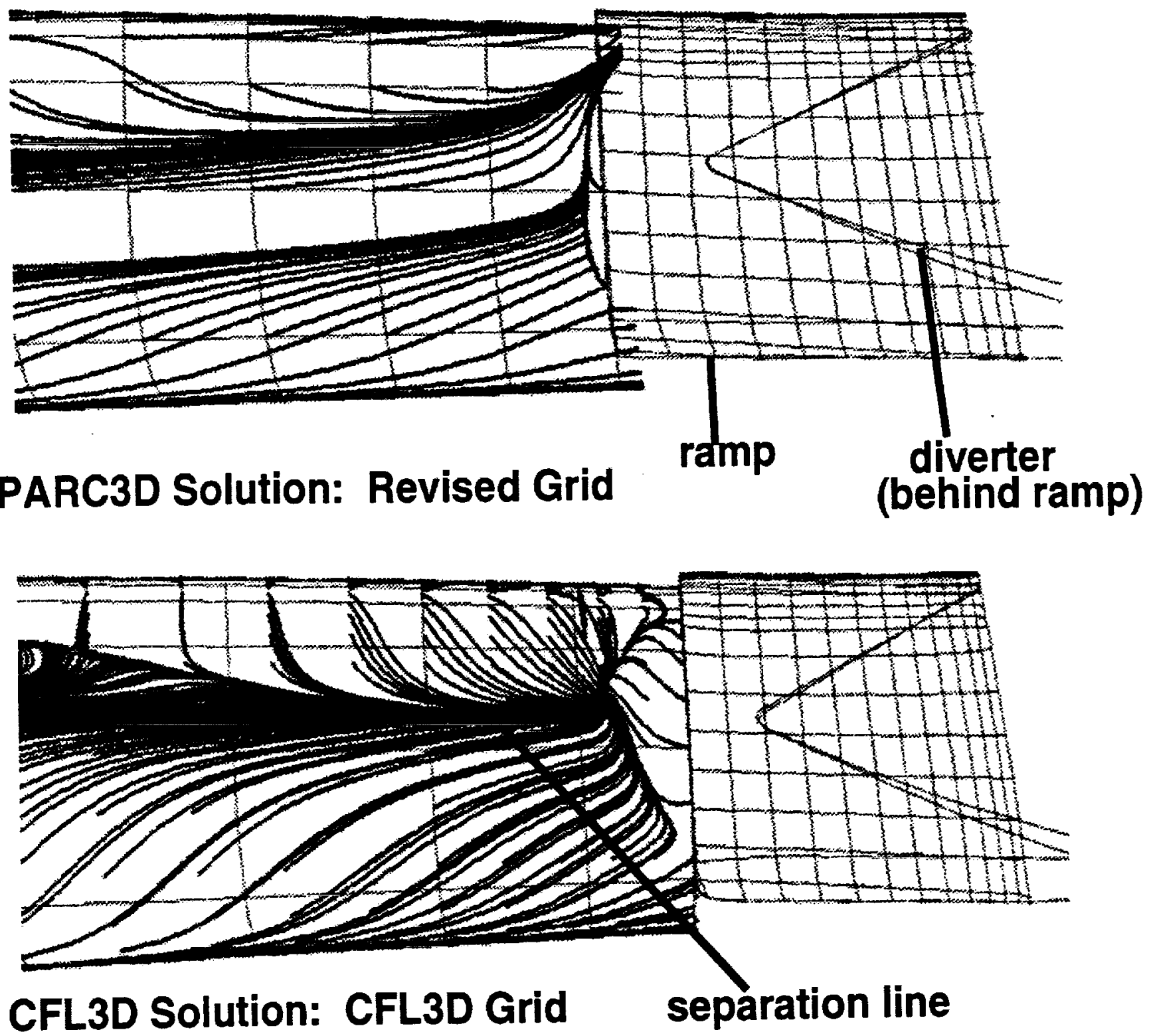

Figure 7. Particle Trajectories Ahead of Ramp 


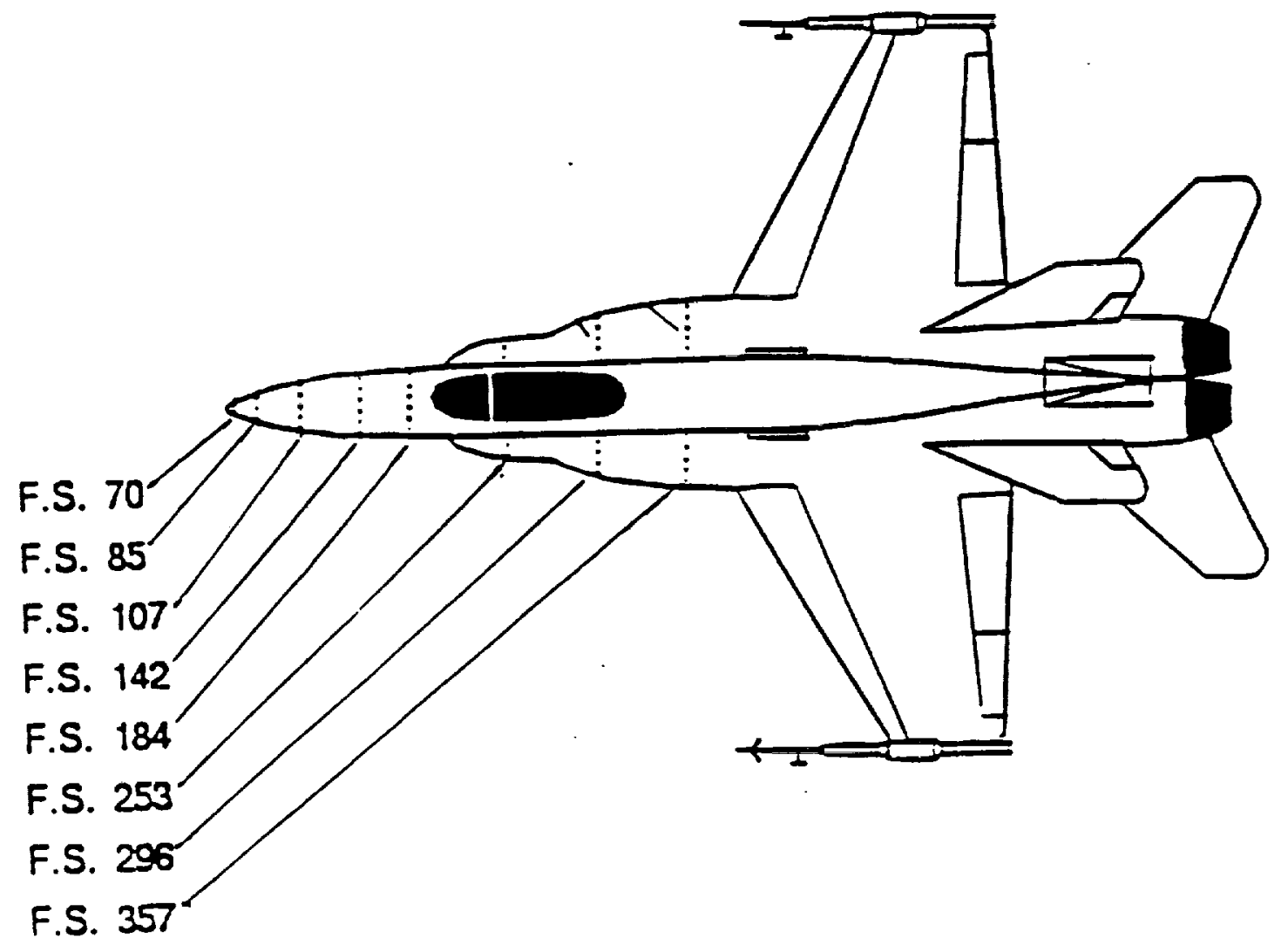

Figure 9. Forebody/LEX Surface Pressure Measurement Stations. 

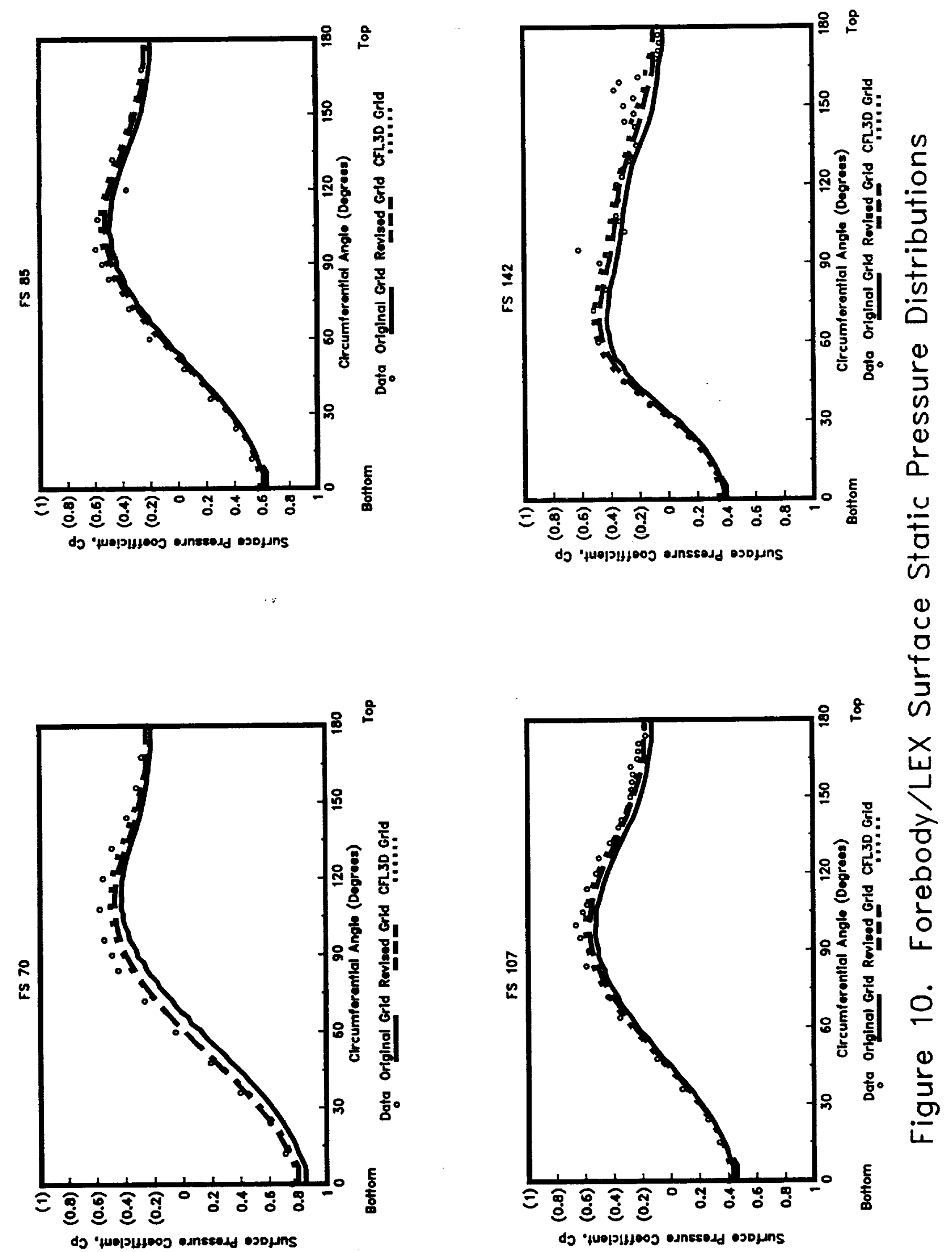

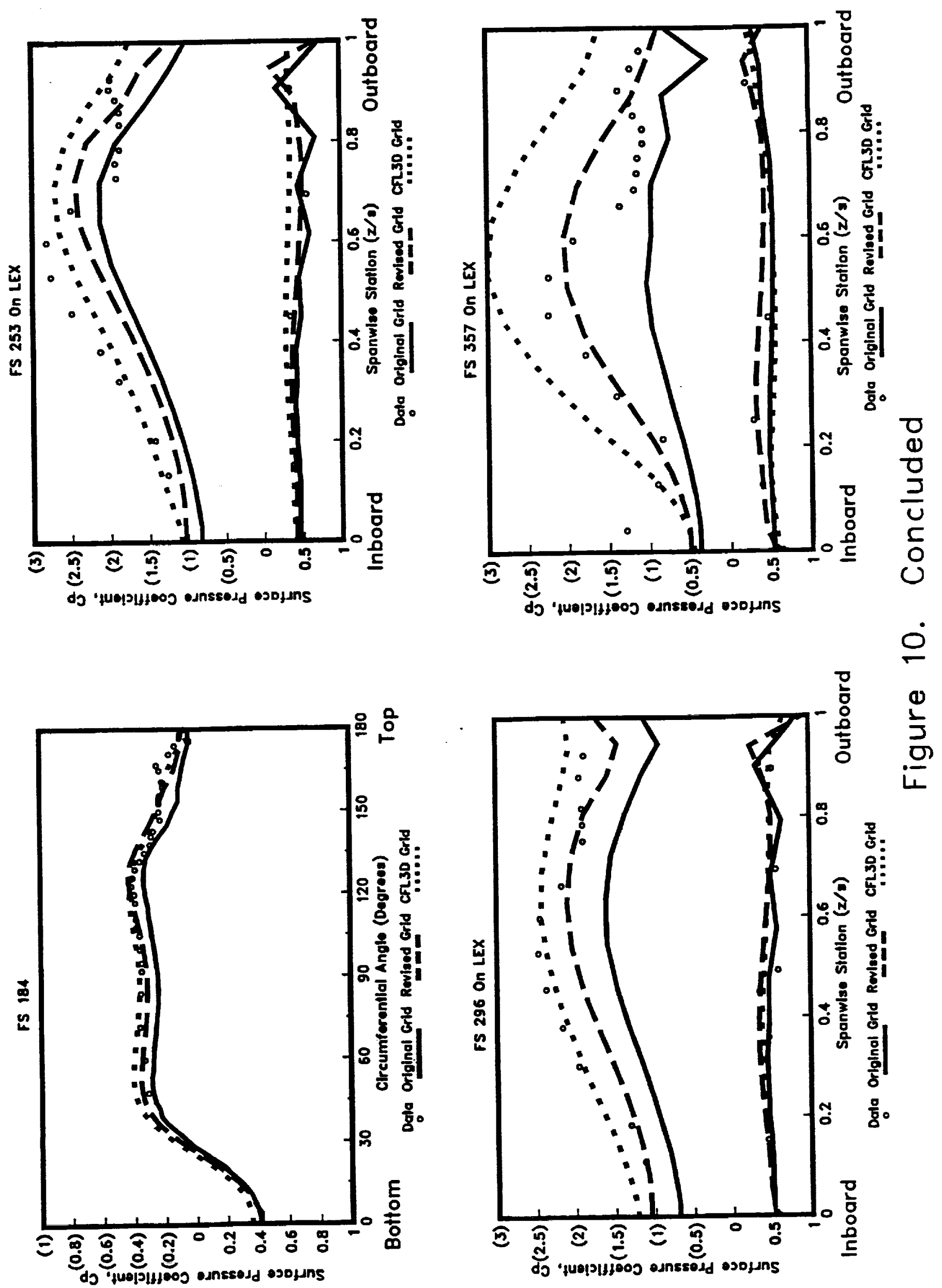


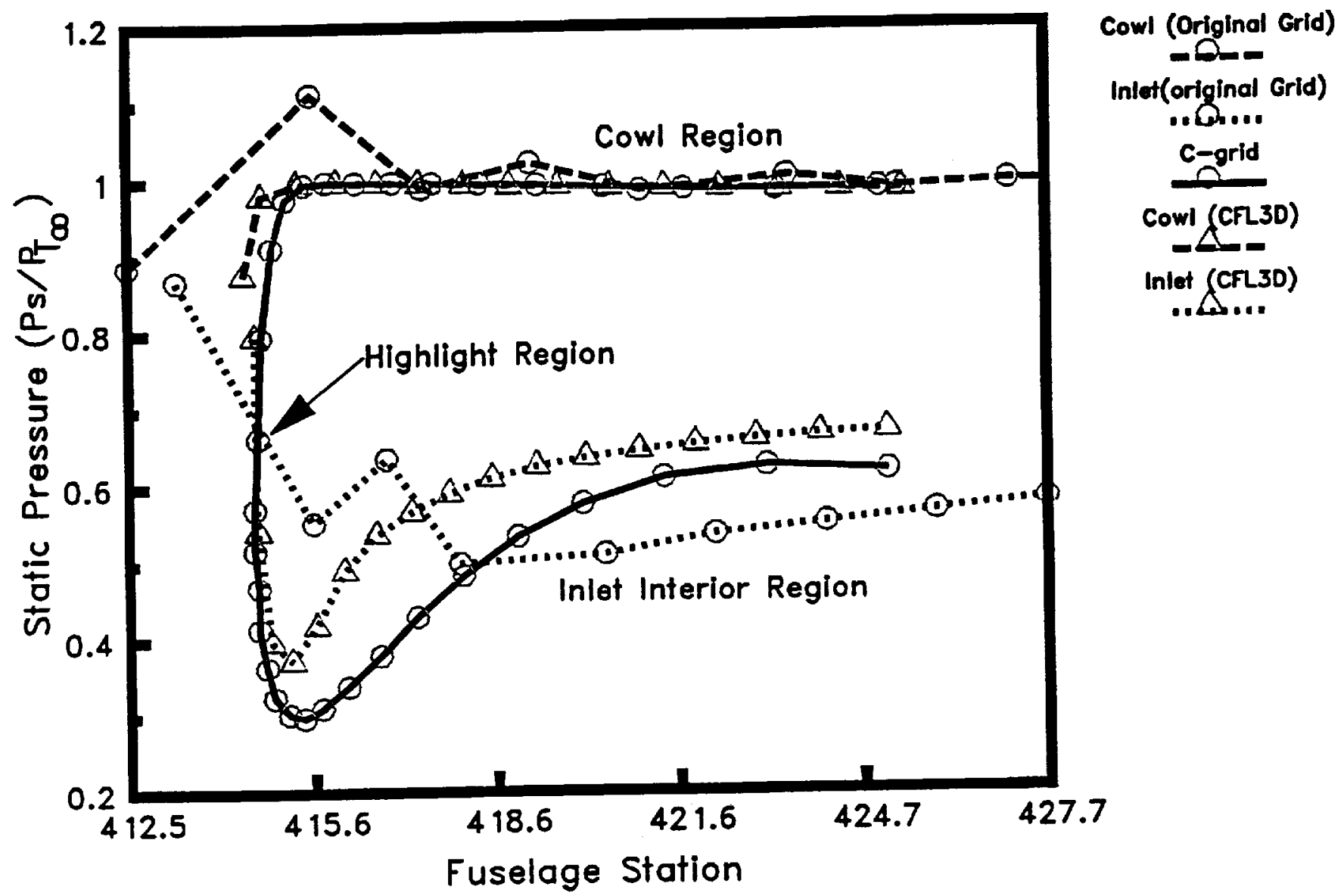

Note: Symbols respresent grid point locations.

Figure 11. Inlet Highlight Surface Static Pressure Distributions at $180^{\circ}$ Circumferential Location (Bottom of Cowl) 

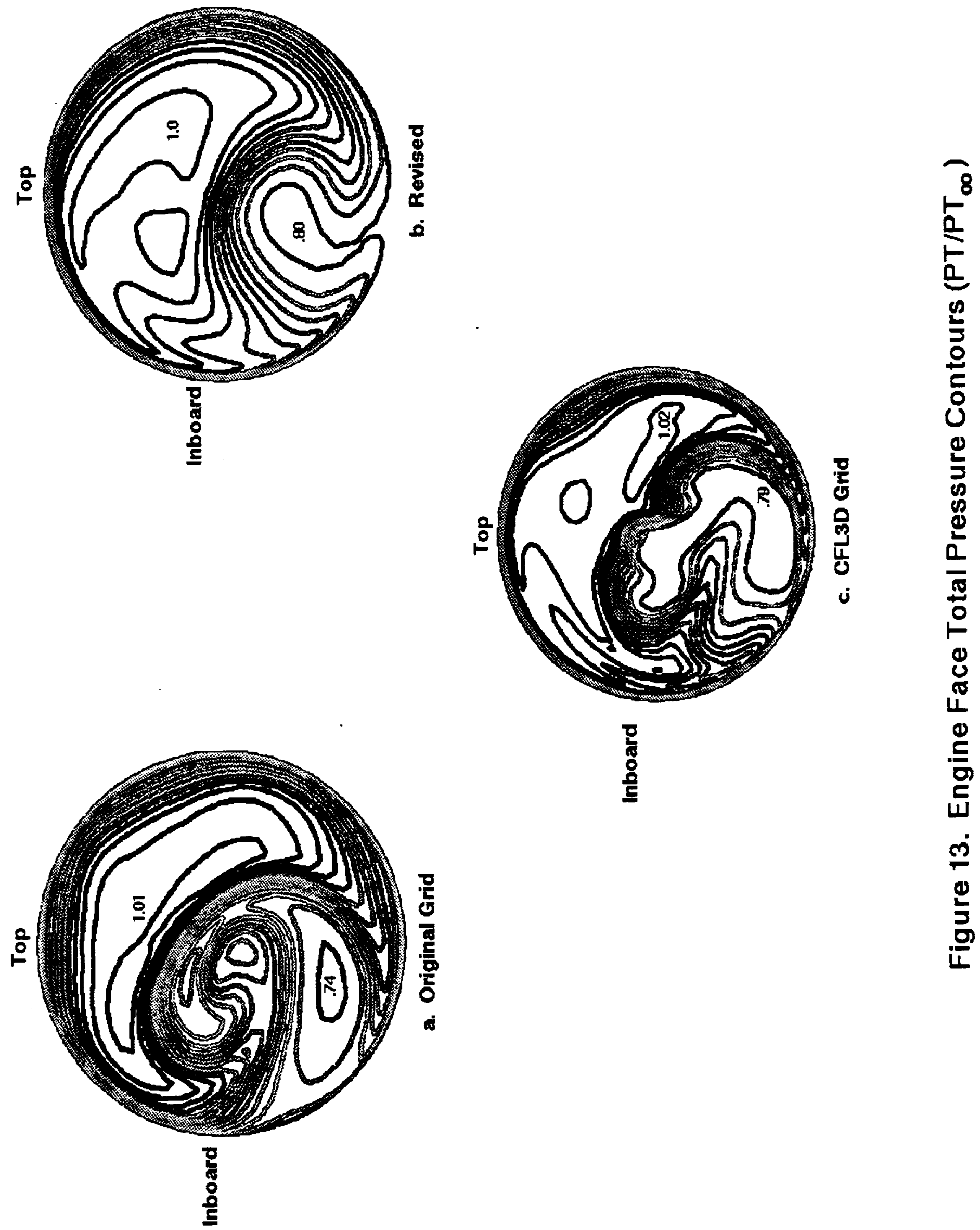

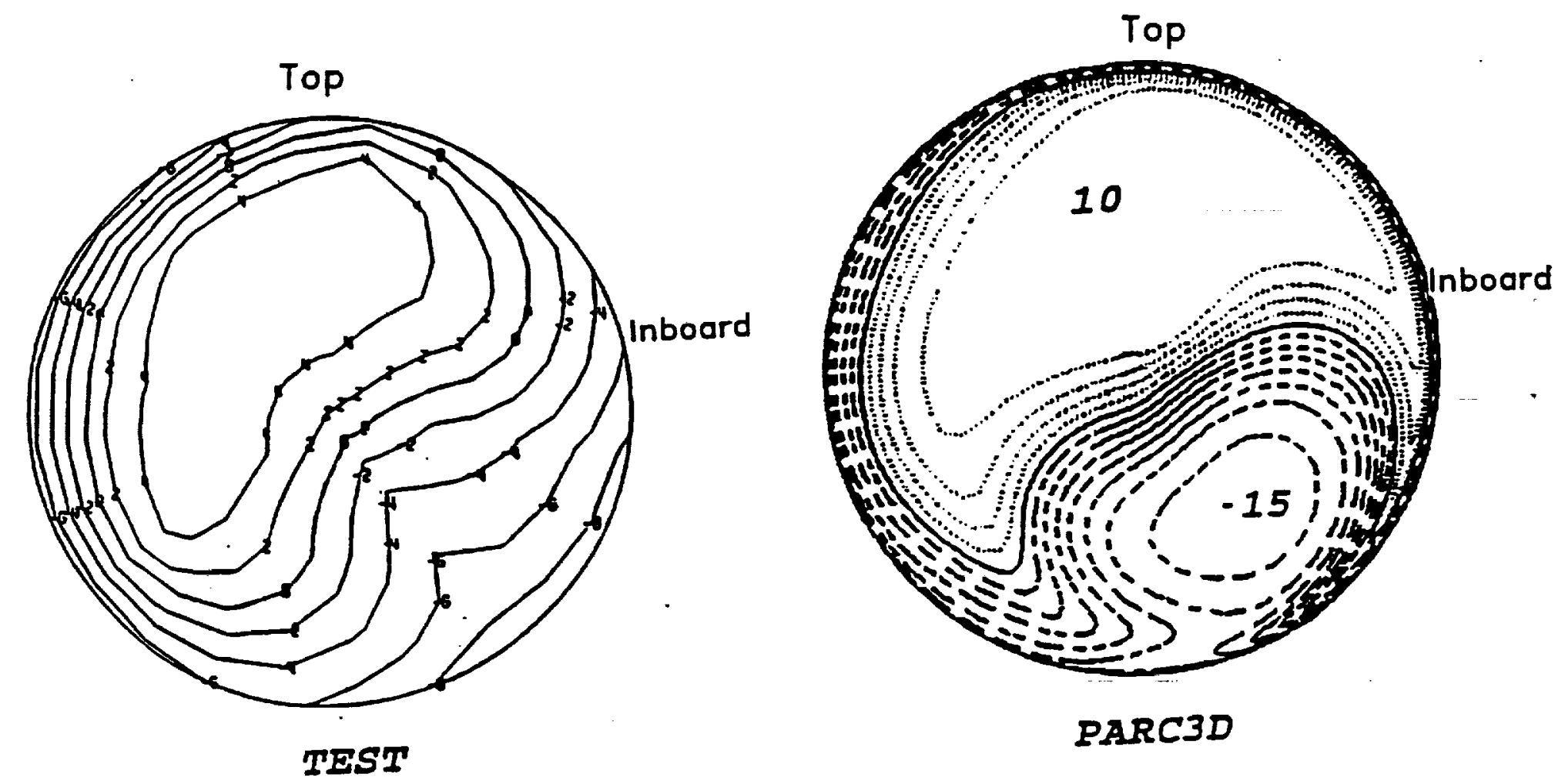

a. Comparison to measured average distortion
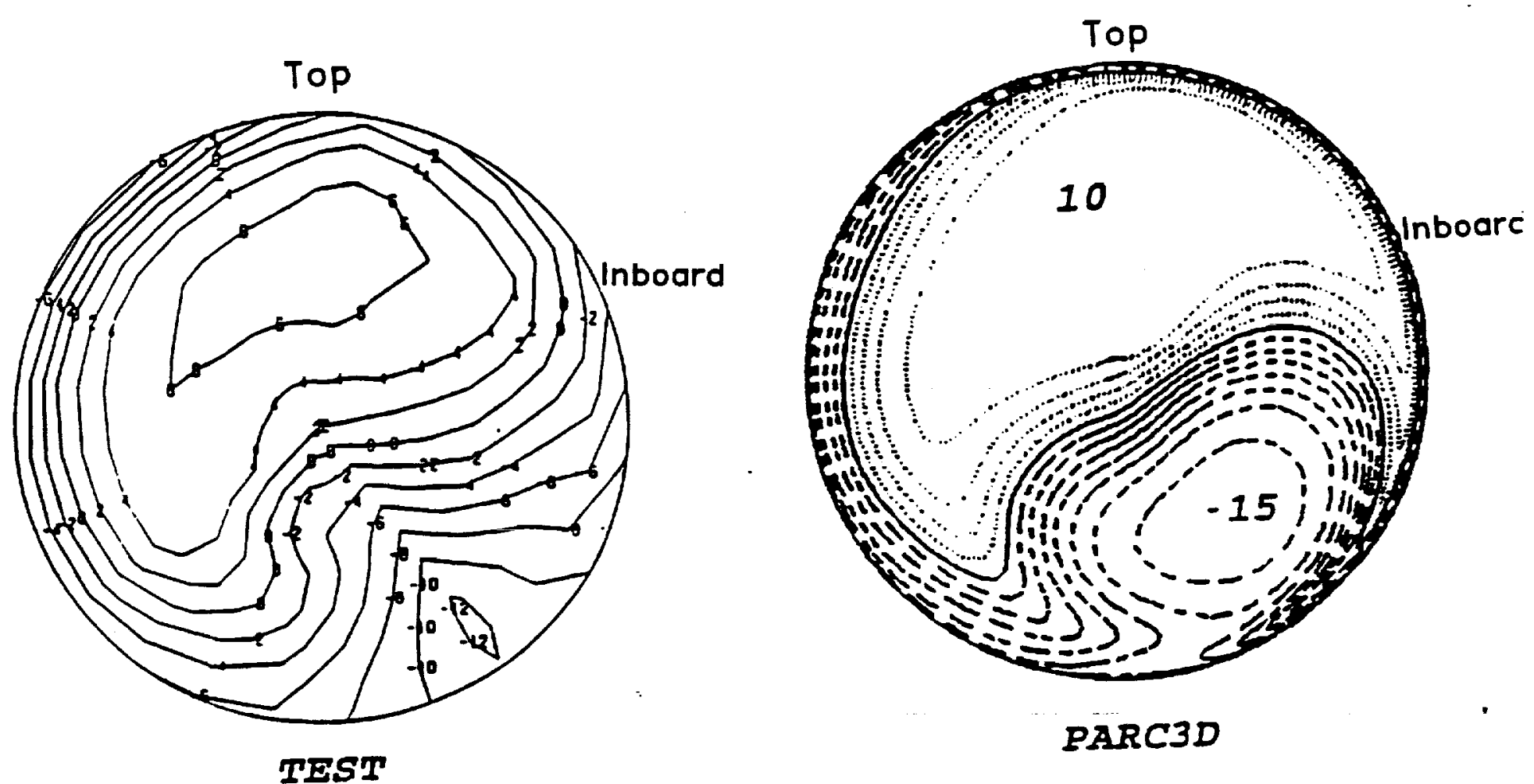

b. Comparison to measured peak distortion

Figure 14. Engine Face Normalized Total Pressure : Contours $\left(\frac{P_{I}-P_{\text {Tavg }}}{P_{\text {Trun }}}\right){ }_{40 .}$ Looking Downstream 

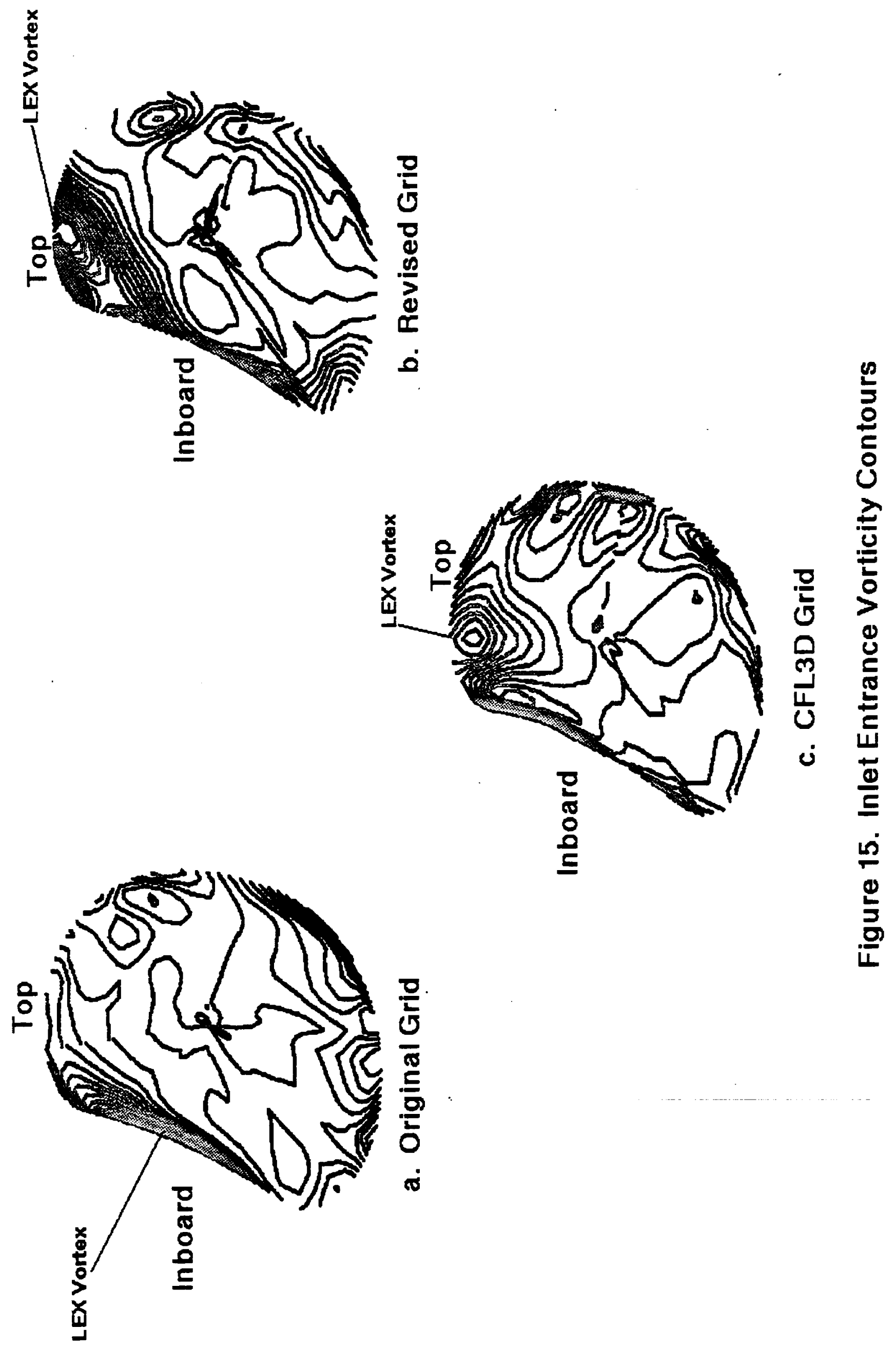

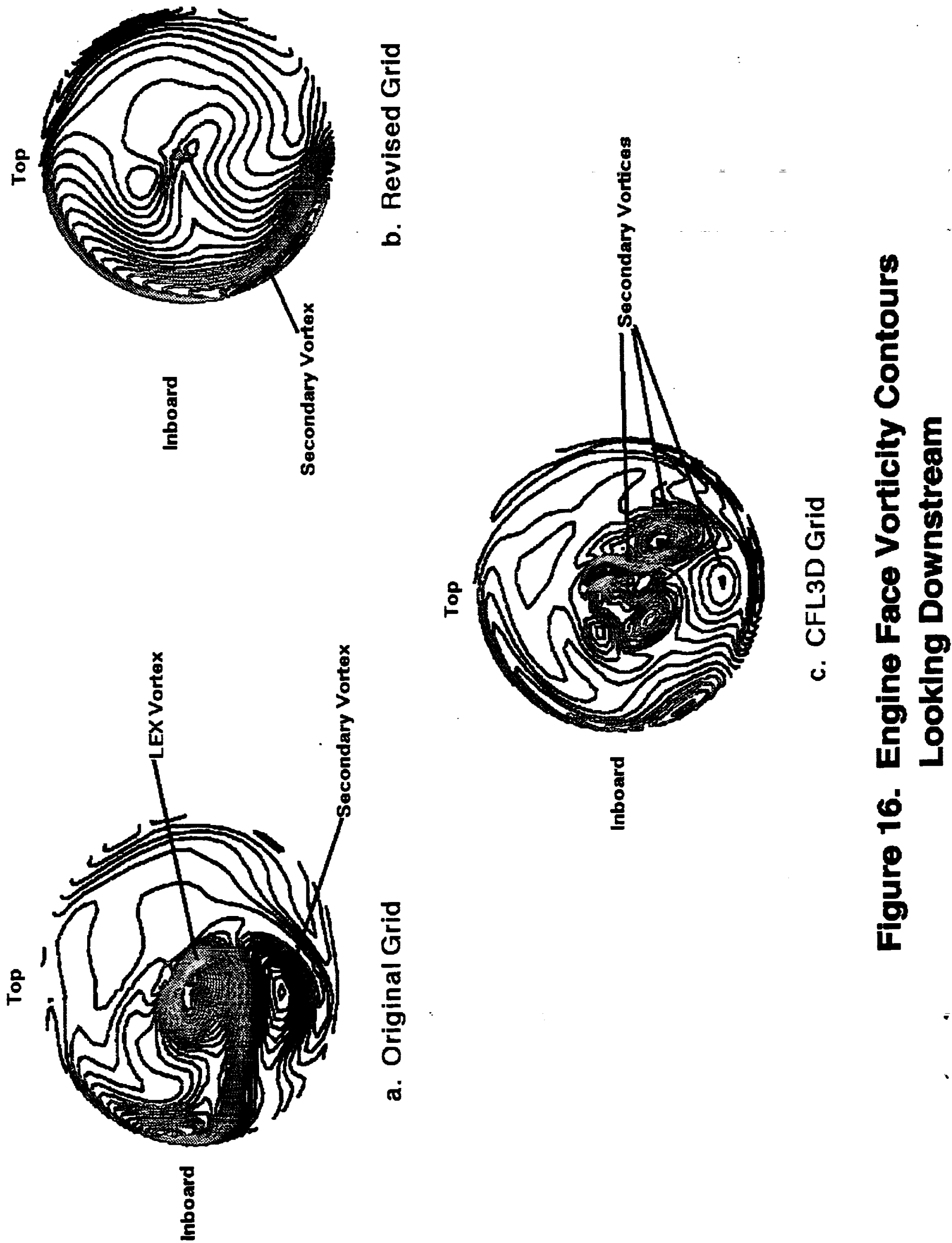

$\frac{0}{\frac{0}{6}}$ 

Public reporting burden for this coltection of information is estimated to average 1 hour per response, including the time for reviewing instructions, searching existing data sources, gathering and maintaining the data needed, and completing and reviewing the collection of information. Send comments regarding this burden estimate or any other aspect of this collection of information, including suggestions for reducing this burden, to Washington Headquarters Services, Directorate for Information Operations and Reports. 1215 Jefferson Davis Highway, Suite 1204. Arlington, VA 22202-4302, and to the Office of Management and Budget, Paperwork Peduction Project (0704-0188), Washington, DC 20503.
1. AGENCY USE ONLY (Leave blank)
2. REPORT DATE
3. REPORT TYPE AND DATES COVERED
April 1994
Final Contractor Report

\section{TITLE AND SUBTITLE}

Installed F/A-18 Inlet Flow Calculations at 30 Degrees Angle-of-Attack:

A Comparative Study

5. FUNDING NUMBERS

\section{AUTHOR(S)}

C. Frederic Smith and Steve D. Podleski

WU-505-68-30

C-NAS3-25266

\section{PERFORMING ORGANIZATION NAME(S) AND ADDRESS(ES)}

Sverdrup Technology, Inc.

Lewis Research Center Group

2001 Aerospace Parkway

Brook Park, Ohio 44142

9. SPONSORINGMONITORING AGENCY NAME(S) AND ADDRESS(ES)

National Aeronautics and Space Administration

Lewis Research Center

Cleveland, Ohio 44135-3191

8. PERFORMING ORGANIZATION REPORT NUMBER

11. SUPPLEMENTARY NOTES

C. Frederic Smith and Steve D. Podleski presently at NYMA, Inc., Engineering Services Division, 2001 Aerospace Parkway, Brook Park, Ohio 44142. Project Manager, Robert E. Coltrin, Propulsion Systems Division, organization code 2780, NASA Lewis Research Center, (216) 433-2181.

12a. DISTRIBUTIONAVAILABILITY STATEMENT 12b. DISTRIBUTION CODE

Unclassified - Unlimited

Subject Category 02

\section{ABSTRACT (Maximum 200 words)}

NASA Lewis is currently engaged in a research effort as a team member of the High Alpha Technology Program (HATP) within NASA. This program utilizes a specially equipped F/A-18, the High Alpha Research Vehicle (HARV), in an ambitious effort to improve the maneuverability of high-performance military aircraft at low-subsonic-speed, high angle-of-attack conditions. The overall objective of the Lewis effort is to develop inlet technology that will ensure efficient airflow delivery to the engine during these maneuvers. One part of the Lewis approach utilizes computational fluid dynamics codes to predict the installed performance of inlets for these highly maneuverable aircraft. Full NavierStokes (FNS) calculations on the installed F/A-18 inlet at 30 degrees angle of attack, 0 degrees yaw and a freestream Mach number of 0.2 have been obtained in this study using an algebraic turbulence model with two grids (original and revised). Results obtained with the original grid were used to determine where further grid refinements and additional geometry were needed. In order to account properly for the external effects, the forebody, Leading Edge Extension (LEX), ramp, and wing were included with inlet geometry. In the original grid, the diverter, LEX slot and leading edge flap were not included due to insufficient geometry definition. These were included in a revised grid and the results obtained with both configurations are presented in this report. In addition, a thin-layer Navier-Stokes (TLNS) code is used with the revised grid and the numerical results are compared to those obtained with the FNS code. The TLNS code was used to evaluate the effects on the solution using a code with more recent CFD developments included such as upwinding with TVD schemes versus central differencing with artificial dissipation. The calculations are compared to a limited amount of available experimental data. the predicted forebody/fuselage surface static pressures compared well with data of all solutions. The predicted forebody/fuselage surface static pressures compared well with data of all solutions. The predicted trajectory of the vortex generated under the LEX was different for each solution. These discrepancies are attributed to differences in the grid resolution and turbulence modelling. All solutions predict that this vortex is ingested by the inlet. The predicted inlet total pressure recoveries are lower than data and the distortions are higher than data. The results obtained with the revised grid were significantly improved from the original grid results. The original grid results indicated the ingested vortex migrated to the engine face and caused additional distortions to those already present due to secondary flow development. The revised grid results indicate that the ingested vortex is dissipated along the inlet duct inboard wall. The TLNS results indicate the flow at the engine face was much more distorted than the FNS results and is attributed to the pole boundary condition introducing numerical distortions into the flow field.

14. SUBJECT TERMS

Inlet; Calculations; F/A-18, Computational fluid dynamics (CFD)

15. NUMBER OF PAGES 51 16. PRICE CODE A04

\begin{tabular}{|c|c|}
\hline $\begin{array}{c}\text { 17. SECURITY CLASSIFICATION } \\
\text { OF REPORT }\end{array}$ & $\begin{array}{c}\text { 18. SECURITY CLASSIFICATION } \\
\text { OF THIS PAGE } \\
\text { Unclassified }\end{array}$ \\
Unclassified
\end{tabular}

NSN 7540-01-280-5500
19. SECURITY CLASSIFICATION OF ABSTRACT

Unclassified
20. LIMITATION OF ABSTRACT

Standard Form 298 (Rev. 2-89)

Prescribed by ANSI Std. Z39-18 298-102 